\title{
Developmental regulation of apical endocytosis controls epithelial patterning in vertebrate
} tubular organs

\author{
Alejo E. Rodríguez-Fraticelli ${ }^{1}$, Jennifer Bagwell ${ }^{2}$, Minerva Bosch-Fortea ${ }^{1}$, Gaelle Boncompain ${ }^{3}$, \\ Natalia Reglero-Real ${ }^{4}$, Maria J. García-León ${ }^{4}$, Germán Andrés ${ }^{5}$, Maria L. Toribio ${ }^{4}$, Miguel A. Alonso ${ }^{4}$, \\ Jaime Millán ${ }^{4}$, Franck Perez ${ }^{3}$, Michel Bagnat ${ }^{2,6}$ and Fernando Martín-Belmonte ${ }^{1,6}$
}

Epithelial organs develop through tightly coordinated events of cell proliferation and differentiation in which endocytosis plays a major role. Despite recent advances, how endocytosis regulates the development of vertebrate organs is still unknown. Here we describe a mechanism that facilitates the apical availability of endosomal SNARE receptors for epithelial morphogenesis through the developmental upregulation of plasmolipin $(p / l p)$ in a highly endocytic segment of the zebrafish posterior midgut. The protein PLLP (PIlp in fish) recruits the clathrin adaptor EpsinR to sort the SNARE machinery of the endolysosomal pathway into the subapical compartment, which is a switch for polarized endocytosis. Furthermore, PLLP expression induces apical Crumbs internalization and the activation of the Notch signalling pathway, both crucial steps in the acquisition of cell polarity and differentiation of epithelial cells. We thus postulate that differential apical endosomal SNARE sorting is a mechanism that regulates epithelial patterning.

To establish functional barriers, epithelial cells require the formation of polarized protein transport machineries ${ }^{1}$. Endocytosis is one of such processes that become highly polarized ${ }^{2}$. Interestingly, recent studies have described that immature epithelial sheets have a reduced rate of apical endocytosis that intensifies along development ${ }^{3}$, which suggests that epithelial cells acquire the ability to internalize material specifically from the apical pole during differentiation. Indeed, apical protein endocytosis regulates polarity and proliferation in Drosophila epithelial cells ${ }^{4,5}$. These findings suggest that endocytosis could be regulated during development to coordinate epithelial morphogenesis ${ }^{6}$. However, the molecular mechanisms of this endocytic regulation in epithelial organ development have not been previously characterized. To unveil developmentally regulated proteins that may control the process of apical endocytosis we used the zebrafish gut morphogenesis model ${ }^{7,8}$. We describe the role of the protein Pllp, which is induced in the posterior segment of the zebrafish intestine during morphogenesis, and is required for the generation of a highly endocytic enterocyte population during gut differentiation. We also characterize the molecular mechanism controlling PLLP function during endocytosis using the three-dimensional (3D)-MDCK model. Using proteomics, we found that in 3D-MDCK cells PLLP interacts with EpsinR (EpsR), an AP1B-binding clathrin adaptor, which regulates the recycling of the endosomal SNAREs. Together, PLLP and EpsR are required for the sorting of endosomal SNAREs into the apical recycling compartment to properly feed the endocytic uptake of apical cargo. Finally, we demonstrate that the endocytic role of PLLP is essential for Crumbs (Crb) downmodulation and Notch activation to promote absorptive cell differentiation.

\section{RESULTS}

PIlp is induced during epithelial tube formation in zebrafish and localizes to a highly endocytic compartment of the midgut Gut morphogenesis is a genetically regulated process. To unveil genes developmentally controlled during epithelial gut morphogenesis we used a screen strategy based on the isolation of epithelial cells from the zebrafish gut $^{8}$. We identified pllp as one of the genes specifically induced during lumen formation and expansion (Fig. 1a). Pllp is a type III transmembrane protein of unknown function that belongs

${ }^{1}$ Department of Development and Differentiation, Centro de Biología Molecular 'Severo Ochoa', CSIC-UAM, Madrid 28049, Spain. ${ }^{2}$ Department of Cell Biology, Duke University, Durham, North Carolina 27710, USA. ${ }^{3}$ Department of Subcellular Structure and Cellular Dynamics, UMR144, Institut Curie, Paris 75005, France.

${ }^{4}$ Department of Immunology and Cell Biology, Centro de Biología Molecular 'Severo Ochoa', CSIC-UAM, Madrid 28049, Spain. ${ }^{5}$ Electron Microscopy Core, Centro de Biología Molecular 'Severo Ochoa', CSIC-UAM, Madrid 28049, Spain.

${ }^{6}$ Correspondence should be addressed to M.B. or F.M-B. (e-mail: m.bagnat@cellbio.duke.edu; fmartin@cbm.csic.es)

Received 24 July 2014; accepted 9 January 2015; published online 23 February 2015; DOI: 10.1038/ncb3106 

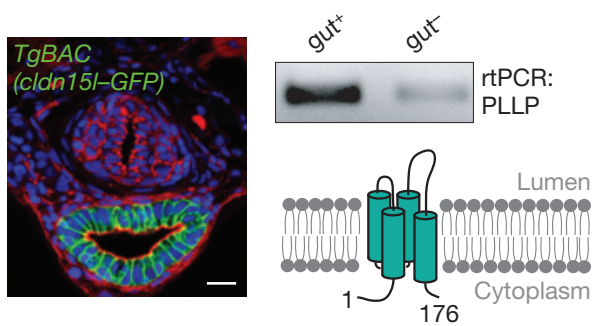

e

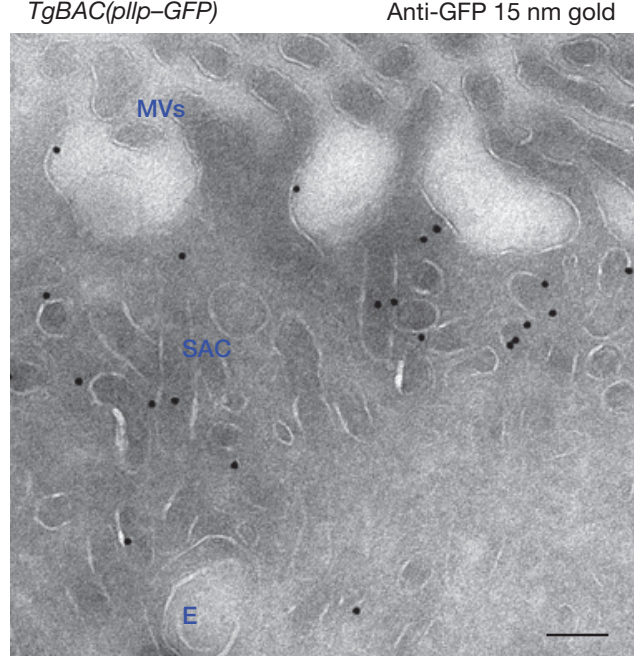

b
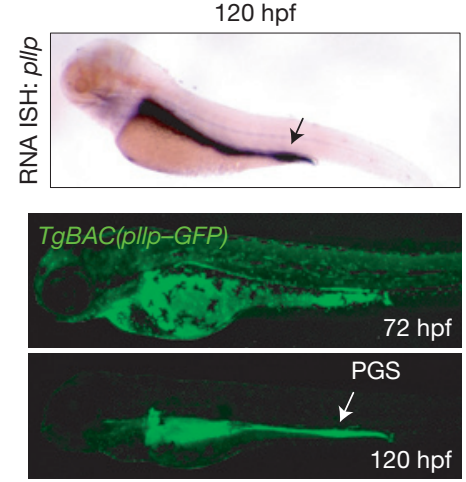

f

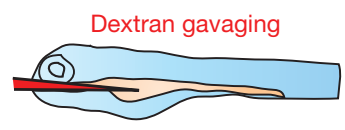

g

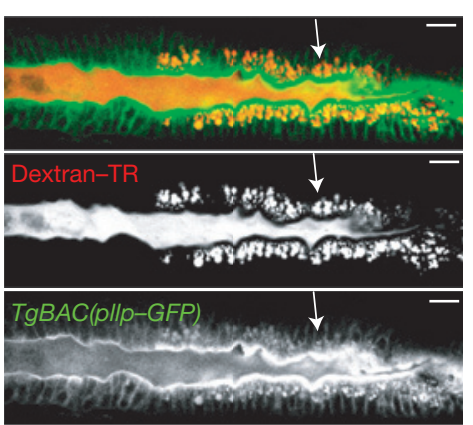

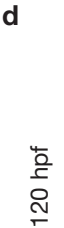
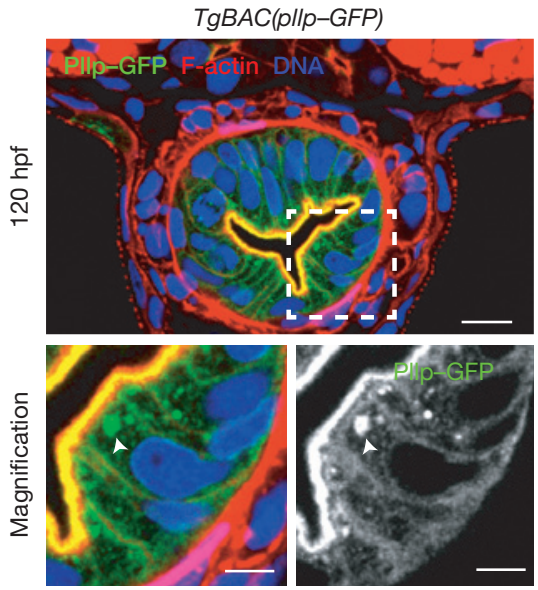

h TgBAC(DIIp-GFP)

TgBAC(lamp2-RFP)

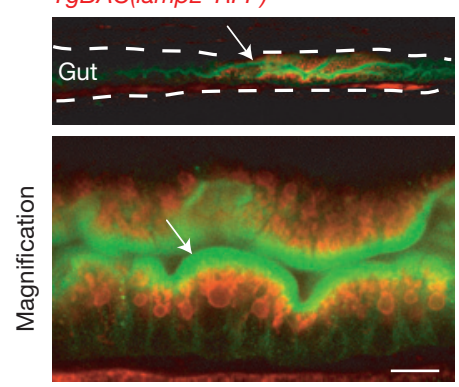

Figure $1 p / l p$ expression is induced in endocytic enterocytes during development. (a) Identification of pllp as a gene induced during gut morphogenesis. Gut cells were FACS-sorted from TgBAC(cldn15/a-GFP) zebrafish larvae and gut-specific cDNAs were cloned by real-time PCR (rtPCR). Bottom right, scheme of predicted PLLP structure showing $\mathrm{N}$ - and C-terminal cytoplasmic tails. Scale bar, $10 \mu \mathrm{m}$. (b) In situ hybridization (ISH) of pl/p DIG-labelled RNA probe at $120 \mathrm{hpf}$. Arrow indicates the gut. (c) TgBAC(p/lp-GFP) transgenic zebrafish larvae. A spacer-GFP sequence was recombined in place of the STOP codon using a zebrafish BAC clone carrying the full pllp gene. Note that the PGS contains a population of PLLPhigh cells (arrow). (d) Transverse section of a TgBAC(p/lp-GFP) larva posterior midgut, stained using phalloidin (which labels F-actin in apical microvilli, in red) and DAPI (for DNA, in blue). Arrowheads indicate apical endosomes. Scale bars, $10 \mu \mathrm{m}$ (magnification, $5 \mu \mathrm{m}$ ). (e) Immunogold

to the family of MARVEL-domain-containing proteins associated with vesicle trafficking and membrane fusion'. We corroborated the expression of pllp in the gut using RNA in situ hybridization (Fig. 1b and Supplementary Fig. 1A). pllp is expressed in the hatching gland and the pronephric duct as early as 48 hours post-fertilization (hpf), and is highly enriched in the gut at $72 \mathrm{hpf}$ and $120 \mathrm{hpf}$ (Fig. 1b, arrows, and Supplementary Fig. 1A). To analyse the subcellular localization of the Pllp protein, we used bacterial artificial chromosome (BAC) recombineering ${ }^{10}$ to generate a BAC expressing Pllp-GFP and obtained stable transgenic animals $T g B A C(p l l p-s p G F P)$. We found that Pllp expression is highly induced in a specific segment of the posterior midgut (PGS) at $120 \mathrm{hpf}$ (Fig. 1c and Supplementary Fig. 1B). Pllp-GFP localizes to the apical region of intestinal epithelial cells (IECs), with a small population associated with internal membranes (Fig. 1d, arrowheads, and Supplementary Fig. 1C). To further evaluate the subcellular localization of the protein, we performed anti-GFP electron microscopy of $T g B A C(p / / p-G F P)$ using anti-GFP and protein-A gold particles. Most labelled protein (65\%) resides in a subapical endosomal compartment (SAC), whereas $15 \%$ of the label localized to microvilli (MVs) and $13 \%$ was labelling more basal localized endosomes (E). Scale bar, $100 \mathrm{~nm}$. (f) Gavaging of zebrafish larvae. Dextran-TR was forcefed by microinjection into anaesthetized $144 \mathrm{hpf}$ larvae. (g) Dextrangavaged $T g B A C(p / / p-G F P)$ larvae. TgBAC(p/lp-GFP) larvae were gavaged with dextran-TR and analysed by live confocal microscopy $2 \mathrm{~h}$ postgavaging. Note that the dextran is endocytosed only in the posterior midgut (arrow), where Pllp expression is higher. Scale bars, $20 \mu \mathrm{m}$. (h) TgBAC(p/lp-GFP); TgBAC(lamp2-spRFP) $144 \mathrm{hpf} \mathrm{larvae} \mathrm{analysed} \mathrm{by} \mathrm{live}$ confocal microscopy. Lamp2 is localized specifically to the posterior midgut (arrows). Scale bar, $10 \mu \mathrm{m}$. Uncropped images of blots/gels are shown in Supplementary Fig. 5.

immunogold electron microscopy in gut sections (Fig. 1e). Most PllpGFP (65\%) localized to small tubules and vesicles (about 70-100 nm wide) present in the first $300 \mathrm{~nm}$ below the apical membrane, with a small fraction of PLLP also distributed both to the apical microvilli and to more basal endosomes. This polarized localization of Pllp suggests a function associated with the apical endocytic pathway.

Therefore, we next analysed whether Pllp is involved in apical endocytosis in the zebrafish gut by using microgavaging to deliver endocytic tracers directly into the intestinal lumen ${ }^{11}$ (Fig. 1f). Interestingly, gavaged dextran-Texas red (TR) was specifically internalized in the posterior midgut in $144 \mathrm{hpf} T g B A C(p l p-s p G F P$ ) larvae where Pllp is enriched (Fig. 1g, arrows). Furthermore, we observed that Lamp2, a late endosomal marker, is specifically enriched in Pllp-positive cells (Fig. 1h, arrows). Thus, Pllp is a marker of highly endocytic enterocytes of the posterior midgut at the onset of intestinal differentiation. 


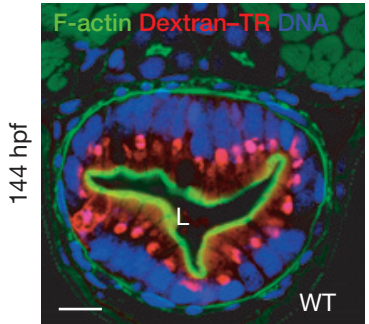

b

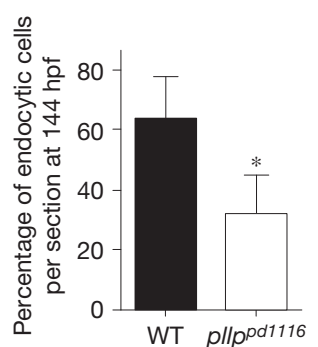

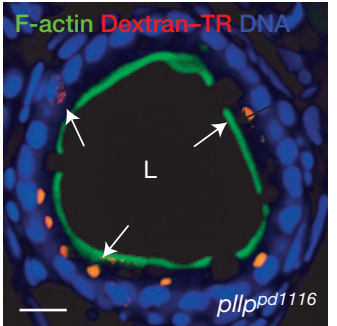

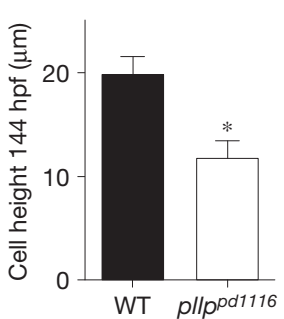

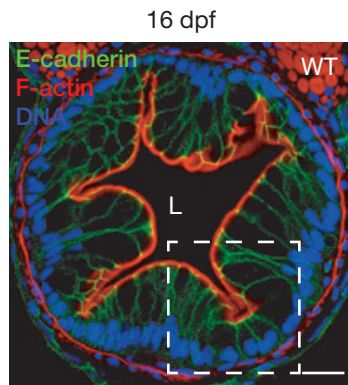

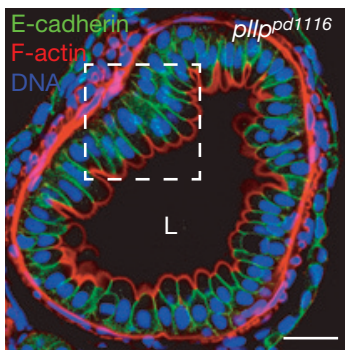

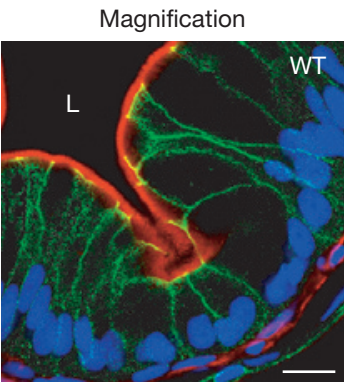

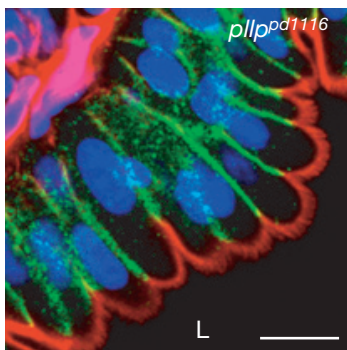

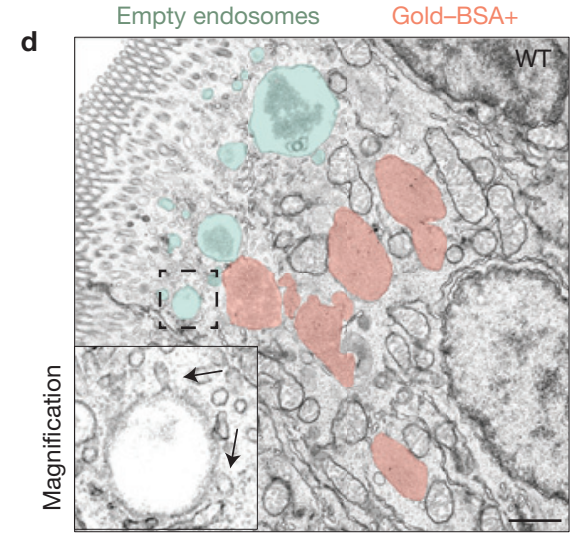
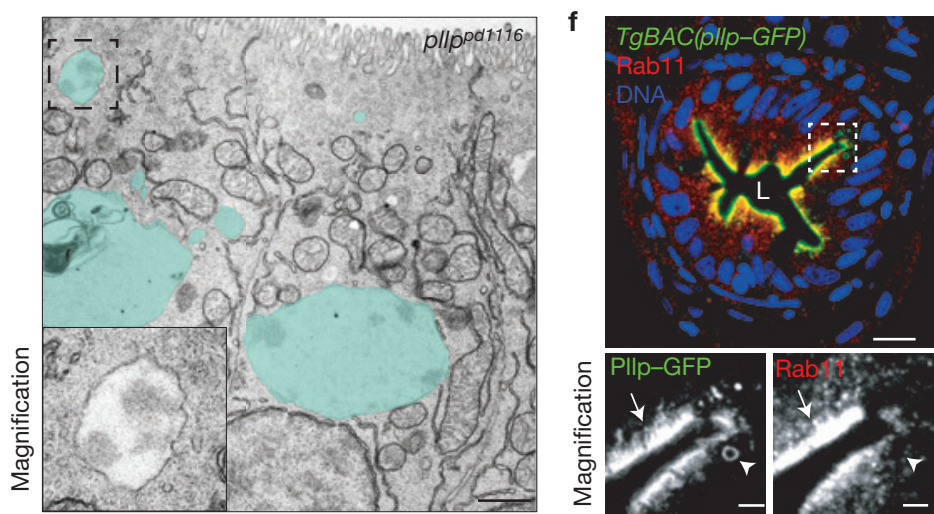

g
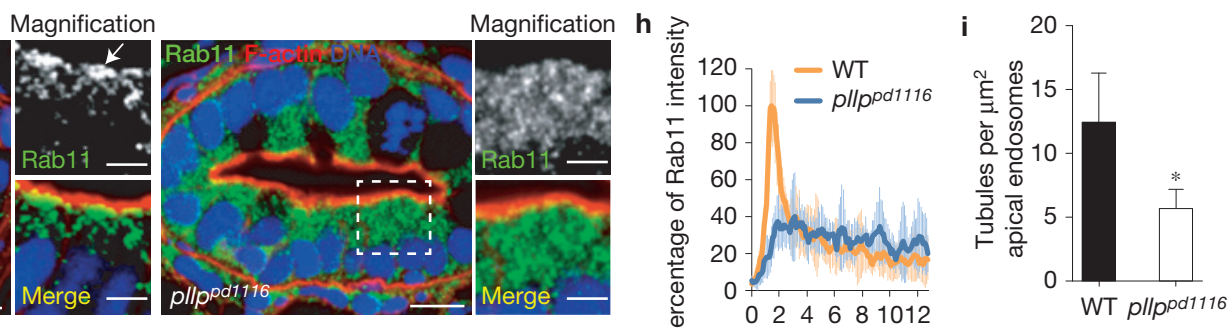

Figure 2 PIIp is required for apical endocytosis and epithelial morphogenesis in the zebrafish gut. (a) Endocytosis of dextran in p/lppd1116 mutants. Posterior midgut sections of dextran-gavaged (red) $144 \mathrm{hpf}$ larvae were labelled with phalloidin (green) and DAPI (blue). Arrows indicate remaining cells that are able to endocytose dextran in the mutant. L, lumen. Scale bar, $10 \mu \mathrm{m}$. (b) Quantification of endocytic cells in p/lppd1116 mutants. Data are mean \pm s.d. percentage of endocytic cells (WT, $63.9 \pm 8.2 \%$; pllp pd1116 $32 \pm 9,3 \% ; n=10$ sections from 5 WT and 6 mutant fish, randomly selected from 3 independent gavaging experiments; ${ }^{*} P<0.005$ (Student's $t$-test)). (c) Quantification of cell height in $p / / p^{\text {pd1116 }}$ mutants. Data are mean \pm s.d. cell height in $\mu \mathrm{m}$ (WT, $19.7 \pm 1.8 \mu \mathrm{m} ; p^{\prime l} p^{p d 116}$, $11.7 \pm 1.7 \mu \mathrm{m} ; n=10$ sections from $7 \mathrm{WT}$ and 6 mutant fish, randomly selected from 3 independent experiments; ${ }^{*} P<0.005$ (Student's $t$-test)). (d) Electron microscopy sections of p/lp pd1116 mutant fish gavaged with dextran and BSA-gold (15 nm). BSA-positive compartments (red) and BSAempty endosomes (green) are coloured. Scale bars, 1 um. (e) Epithelial morphology is disrupted in p/lp pd1116 juveniles. Larvae were raised in 1 I tanks, fixed at $16 \mathrm{dpf}$, sectioned and stained with the anti-E-cadherin antibody (green), phalloidin (red) and DAPI (blue). L, Iumen. Scale bars,
$20 \mu \mathrm{m}$ (magnification, $10 \mu \mathrm{m}$ ). (f) PLLP and Rab11a co-localization in zebrafish enterocytes. TgBAC(p/lp-GFP) $144 \mathrm{hpf}$ guts are labelled with antiRab11 (red) and DAPI (blue). Arrows indicate co-localization. Arrowheads indicate Rab11-negative PLLP endosomes. L, lumen. Scale bars, $10 \mu \mathrm{m}$ (magnification, $5 \mu \mathrm{m}$ ). (g) Rab11 localization in WT and p/lp pd1116 $96 \mathrm{hpf}$ larval guts. Larvae were fixed, sectioned and stained with anti-Rab11 (green), phalloidin (red) and DAPI (blue). Arrow indicates the subapical compartment. Scale bars, $10 \mu \mathrm{m}$ (magnification, $5 \mu \mathrm{m}$ ). (h) Quantification of Rab11 localization in $\mathbf{g}$. Fluorescent intensity linear profiles were drawn perpendicular to the centre of the apical plasma membrane ( 0 is the peak of apical F-actin staining). Data are averaged linear profiles \pm s.d. ( $n=16$ WT and 12 mutant cells from 3 independent experiments). (i) Quantification of tubulating membranes in WT and p/lp pd1116 larvae. The number of connected tubular structures was counted in every apical endosome $(>300 \mathrm{~nm}$ diameter). For every endosome, the endosomal surface was determined as a function of perimeter $\times$ slice depth. Results are represented as number of tubules counted per endosomal surface unit (in $\left.\mu \mathrm{m}^{2}\right) \pm$ s.d. $(n=5$ WT and 5 mutant fish pooled from 2 independent experiments; ${ }^{*} P<0.05$ (Student's $t$-test)). 


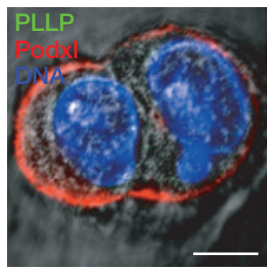

b

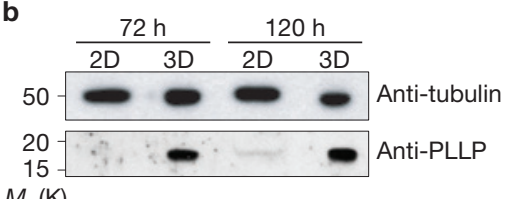

$M_{\mathrm{r}}(\mathrm{K})$

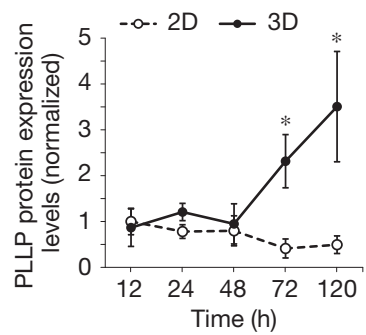

$20 \mathrm{~h}$

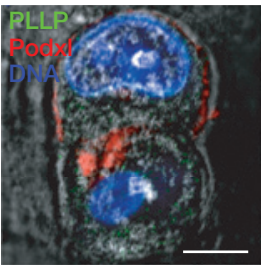

$36 \mathrm{~h}$

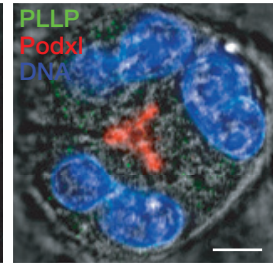

$72 \mathrm{~h}$

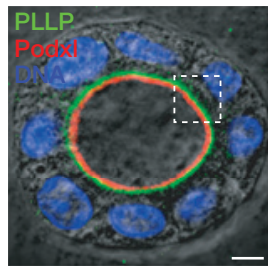

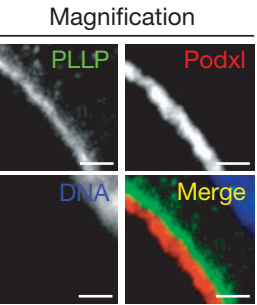

C
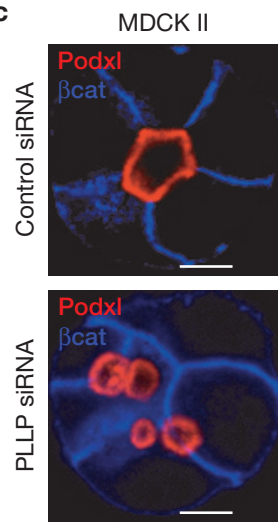
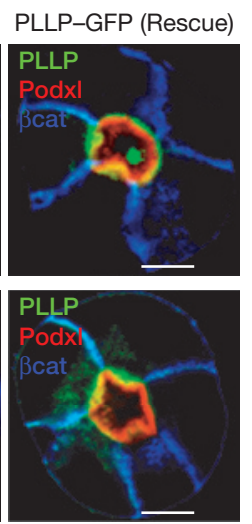
SiRNA: $-\quad+\quad++$ WB: Anti-PLLP
Rescue: $-\quad+\quad+$ WB:

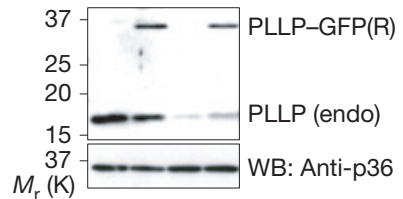

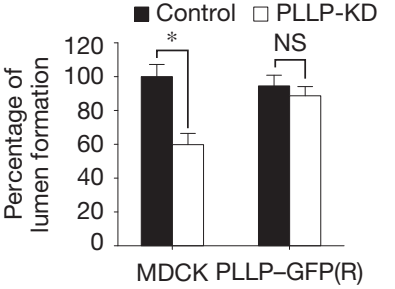

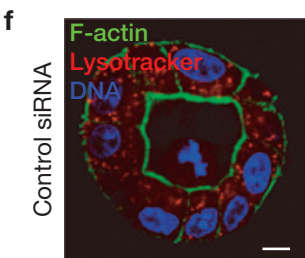
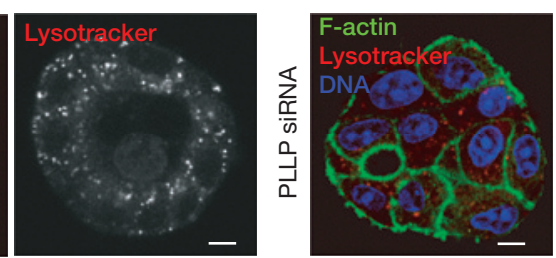
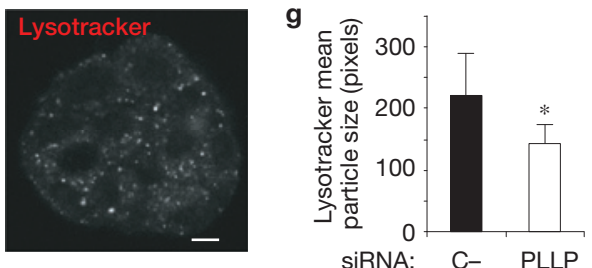
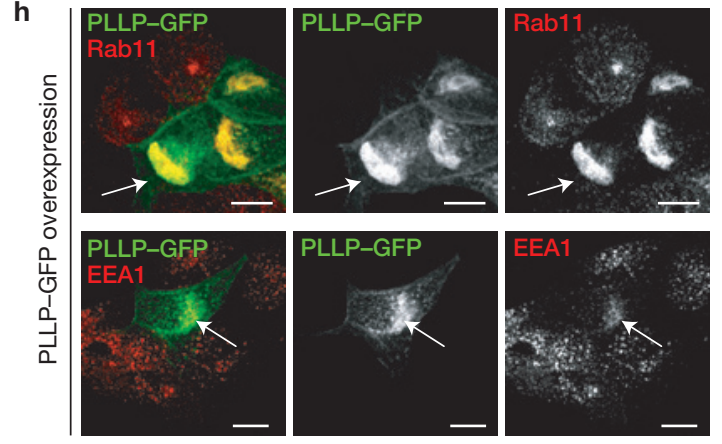

Figure 3 PLLP is required for epithelial morphogenesis and endosomal maturation in MDCK cysts. (a) Expression of PLLP in MDCK cysts at different time points. MDCK cells were grown to form cysts and fixed after $12,20,36$ and $72 \mathrm{~h}$. MDCK cysts were labelled with anti-PLLP antibody (green), anti-Podxl (red) and DNA (blue) and analysed by confocal microscopy using differential interference contrast. Scale bars, $5 \mu \mathrm{m}$ (magnification, $2 \mu \mathrm{m})$. (b) Expression of PLLP in MDCK cysts and monolayers at different time points. MDCK cells were grown to form cysts and lysed after $12,24,48,72$ and $120 \mathrm{~h}$. Western blot analysis was performed to quantify PLLP protein levels at different time points (bottom graph). Data represent mean \pm s.d. $\left(~ n=4\right.$ independent western blots; ${ }^{*} P<0.005$ (Student's $t$-test); statistics source data can be found in Supplementary Table 3). (c) PLLP-KD phenotype in MDCK cysts, and phenotype rescue. WT MDCK cells or MDCK cells stably expressing siRNA-resistant PLLP-GFP(R) protein were transfected with control or PLLP-specific siRNAs and grown to form cysts. MDCK cysts were fixed and labelled with anti-Podxl (red), anti- $\beta$ - catenin (blue) and analysed by confocal microscopy. Scale bars, $5 \mu \mathrm{m}$. (d) Western blot of PLLP KD and rescue experiments. Whole-cell lysates were prepared and analysed by western blotting using anti-PLLP antibody and anti-p36 as a loading control. (e) Quantification of PLLP-KD
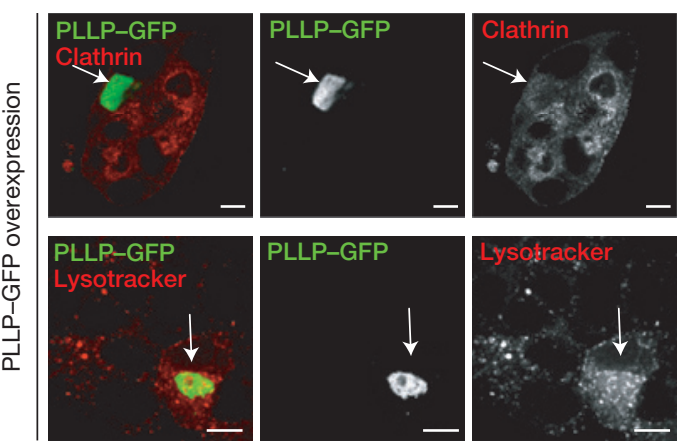

phenotype and rescue. Measurements are normalized to WT MDCK cells (control) and expressed as mean \pm s.d. percentage relative to control singlelumen-forming cysts (control, $100 \pm 7.2 \%$, siRNA PLLP, 59.8 $\pm 6.6 \%$, PLLP(R)-control, $94.5 \pm 6.3 \%$, PLLP(R)-siRNA PLLP, $88.7 \pm 5.4 \% ; n=3$ independent transfection experiments; ${ }^{*} P<0.005$, NS, not significant (Student's $t$-test); statistics source data can be found in Supplementary Table 3). (f) Endosomal acidification defect in PLLP-KD cysts. MDCK cells transfected with control or PLLP-specific siRNAs were grown to form cysts for $72 \mathrm{~h}$, labelled with Lysotracker-red for $2 \mathrm{~h}$, and then fixed. MDCK cysts were also labelled with phalloidin (green) and ToPRO3 (DNA, blue) and analysed by confocal microscopy. Scale bars, $5 \mu \mathrm{m}$. (g) Quantification of endosomal acidification defect in $\mathbf{f}$. Data are represented as mean \pm s.d. particle size in square pixels $(n=17$ control and 18 PLLP-KD cysts selected randomly from 4 independent siRNA experiments; ${ }^{*} P<0.01$ (Student's $t$-test)). (h) Overexpression of PLLP-GFP in MDCK monolayers. MDCK cells transiently transfected with PLLP-GFP (green) were analysed at $48 \mathrm{~h}$ and labelled with anti-Rab11, anti-EEA1, anti-Clathrin and Lysotracker (red). Arrows indicate endosomal aggregates in PLLPoverexpressing cells. Scale bars, $5 \mu \mathrm{m}$. Uncropped images of blots/gels are shown in Supplementary Fig. 5. 


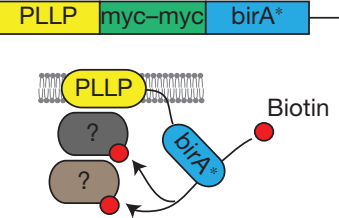

b EpsinR:

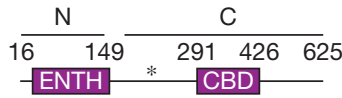

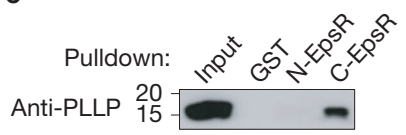

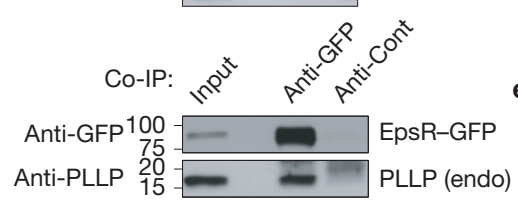

Anti-EpsR 75

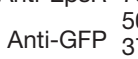

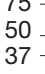

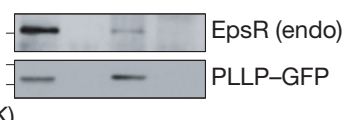
$M_{\mathrm{r}}(\mathrm{K})$

d

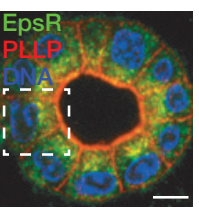

e

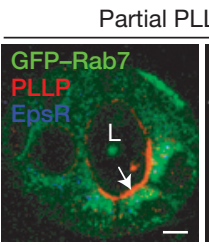

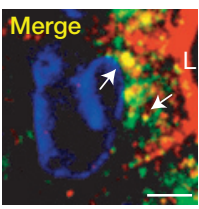

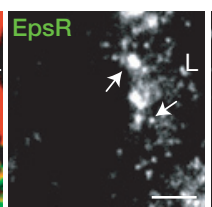

f Control siRNA
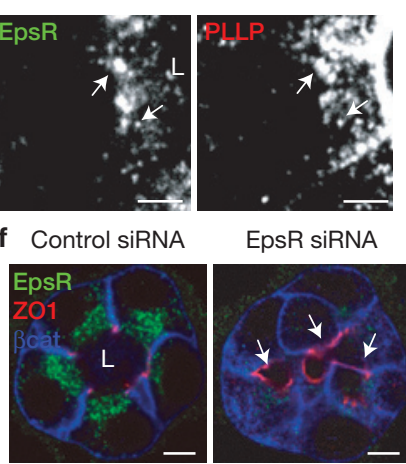

EpsR siRNA
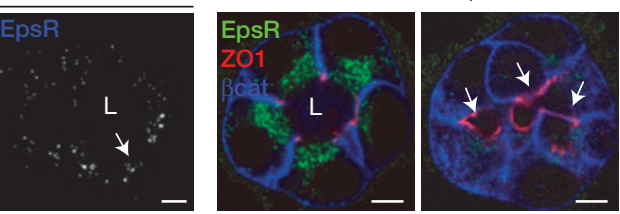

i
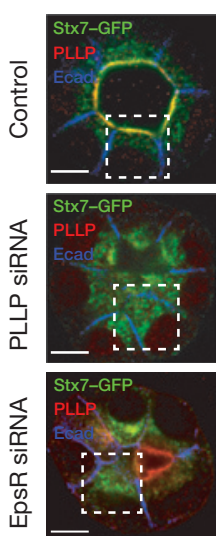

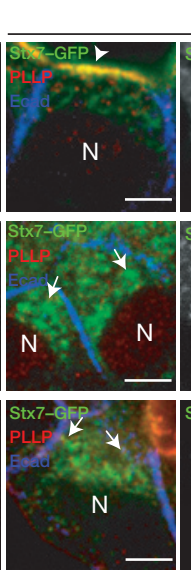

Magnification
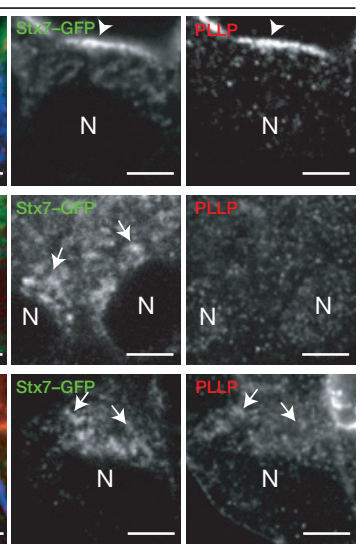

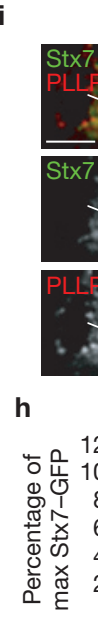

Bleaching Fluorescence recovery

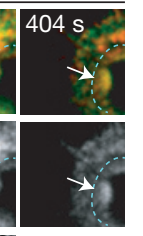

k

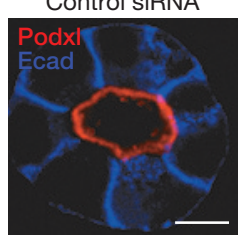

Stx7 siRNA

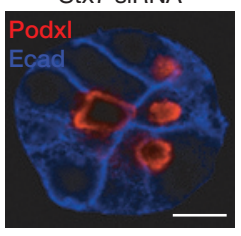

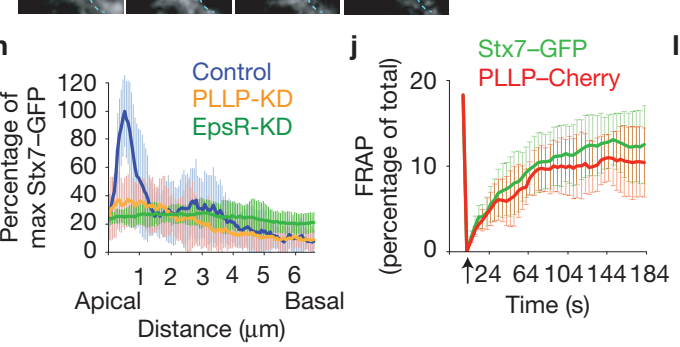

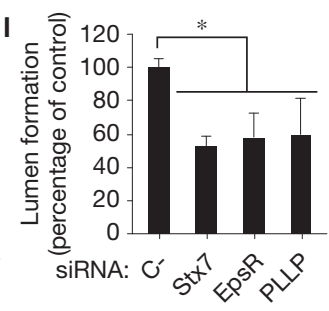

Figure 4 EpsR/PLLP mediate polarized apical sorting of endosomal SNARE Stx7. (a) In vivo biolD assay to identify PLLP-interacting proteins. (b) Protein structure of EpsR. EpsR was biotinylated in a specific segment of the C-terminal region (asterisk). (c) Pulldown and co-immunoprecipitation of endogenous PLLP and EpsR using GST-tagged EpsR fragments, EpsR-GFP or PLLP-GFP. (d) Endogenous EpsR and PLLP localization. MDCK cysts were labelled with anti-PLLP antibody (red), anti-EpsR (green) and DNA (blue). Arrows indicate PLLP and EpsR co-localizing in perinuclear endosomes. Scale bars, $5 \mu \mathrm{m}$ (magnification, $2 \mu \mathrm{m}$ ). (e) Disrupted Rab7 and EpsR localization in PLLP-KD. MDCK cells stably expressing Rab7-GFP were transfected with PLLP siRNAs, grown in cysts and labelled with anti-PLLP (red) and anti-Epsin-R (blue). Arrow indicates non-depleted cells. Scale bars, $5 \mu \mathrm{m}$. (f) Phenotype of EpsR-KD. MDCK cells were transfected with control or EpsRspecific siRNAs, grown in cysts and labelled with anti-EpsR (green), anti-ZO1 (red) and anti- $\beta$ catenin (blue). L, lumen. Arrows indicate multiple lumina. Scale bars, $5 \mu \mathrm{m}$. (g) Stx7 localization in PLLP-KD and EpsR-KD MDCK cysts. MDCK cells stably expressing Stx7-GFP were transfected with control, PLLP or EpsR-specific siRNAs, grown in cysts and labelled with anti-PLLP (red) and anti-E-cadherin (blue). Arrowheads indicate PLLP/Stx7 co-localization. Arrows indicate PLLP/Stx7 in perinuclear endosomes. Scale bars, $5 \mu \mathrm{m}$. (h) Quantification of Stx7 localization in g. GFP-Stx7 linear profiles were drawn perpendicular to the centre of the apical plasma membrane. Data represented are averaged linear profiles \pm s.d. ( $n=19$ control and 27 PLLPKD cells randomly selected from 4 independent experiments). (i) Stx7 and PLLP FRAP assay. MDCK cells expressing PLLP-Cherry were transfected with Stx7-GFP and grown in cysts for $72 \mathrm{~h}$. Photobleaching was performed inside the region outlined by the dashed line, and cysts were imaged every $4 \mathrm{~s}$. Scale bars, $5 \mu \mathrm{m}$. (j) Quantification of FRAP assay. Data are mean \pm s.d. percentage of total fluorescence intensity inside the photobleached region ( $n=6$ cysts from 3 independent FRAP experiments). (k) Phenotype of Stx7-KD. MDCK cells transfected with control or Stx7-specific siRNAs were grown in cysts and labelled with anti-Podxl (red) and anti-E-cadherin (blue). Scale bars, $5 \mu \mathrm{m}$. (I) Quantification of phenotypes in PLLP-KD, EpsR-KD and Stx7-KD MDCK cysts. Measurements are expressed as mean \pm s.d. percentage (relative to control) of single-lumen-forming cysts in 3 different independent experiments (control, $100 \pm 5.3 \%$; Stx7-KD, $52.3 \pm 6.4 \%$; EpsR-KD, 58.06 $\pm 14.5 \% ;$ PLLP-KD, $59.7 \pm 22.1 \% ; n=3$ independent transfection experiments; ${ }^{*} P<0.005$ (Student's $t$-test), statistics source data can be found in Supplementary Table 3). Uncropped images of blots/gels are shown in Supplementary Fig. 5.

\section{Pllp is required for apical endocytosis and endosomal maturation in the zebrafish posterior midgut}

To analyse whether Pllp is required for apical endocytosis in the gut, we generated a mutant allele using TAL-effector nucleases ${ }^{12}$ (TALENs). We identified one allele that contained an insertion/deletion ( $p d 1116)$, giving rise to a frame-shift mutation and an early STOP codon, which truncates $85 \%$ of the protein structure (Supplementary Fig. 1D). Homozygous pllp pd1116 larvae develop normal early gut morphology and intestinal cell numbers (Supplementary Fig. 1E,F), but present marked defects in the number of endocytic cells and the amount of dextran that was internalized in the PGS (Fig. 2a,b). In addition, at $144 \mathrm{hpf}$, pllp pd1116 IECs are significantly shorter than the wild type (WT; Fig. 2c and Supplementary Fig. 1G), a phenotype also observed in pllp morphants (Supplementary Fig. 1H-J), and present stubbier microvilli (Supplementary Fig. 1K). To more precisely evaluate the internalization defects, we gavaged $p l l p^{p d 1116}$ larvae with dextran-TR 

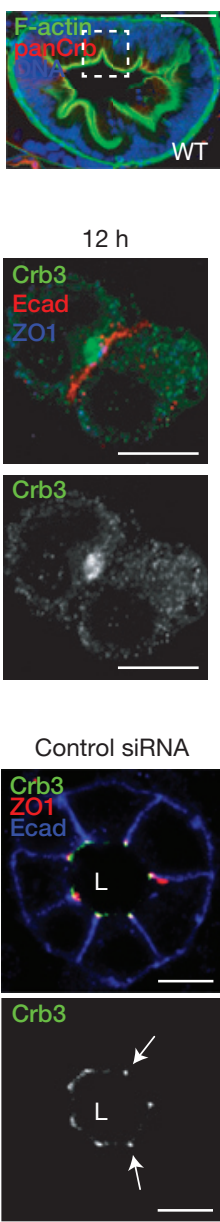

g

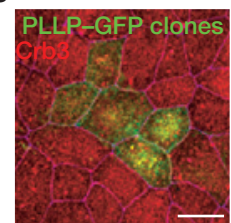

Magnification

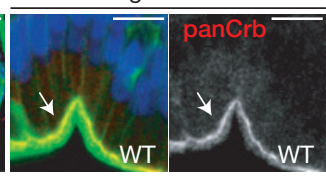

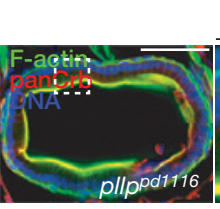

$16 \mathrm{~h}$
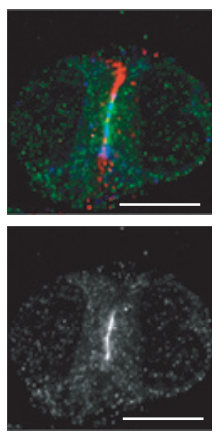

PLLP siRNA
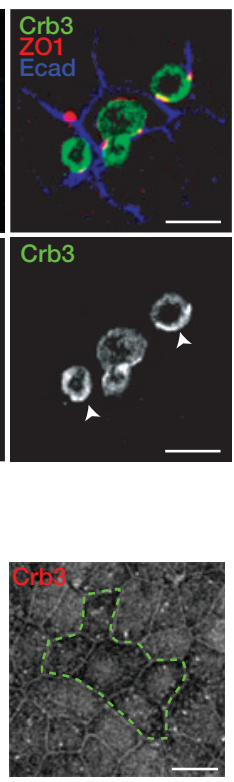
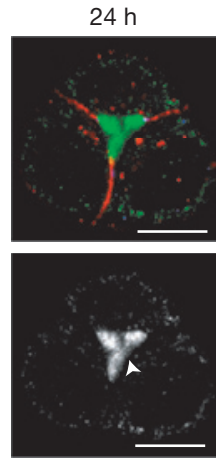

Stx7 siRNA
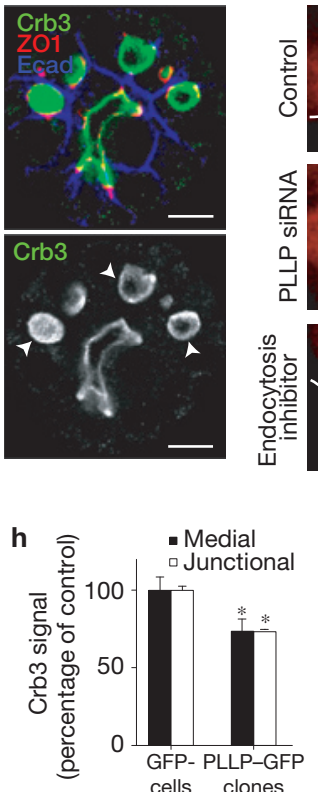

$48 \mathrm{~h}$
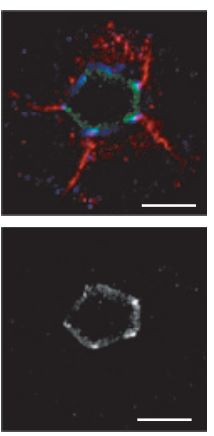

f GFP-Crb3 RUSH
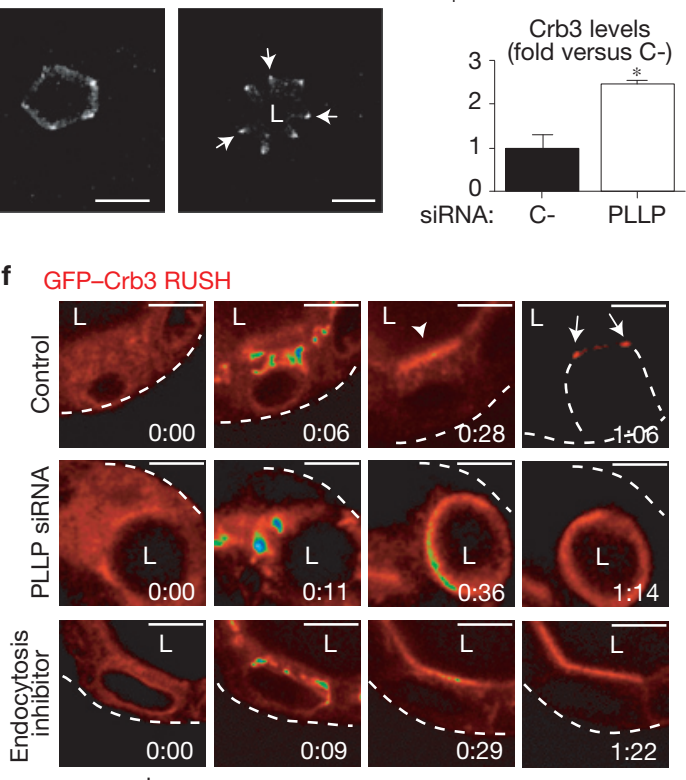

PLLP

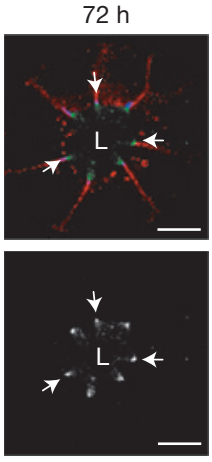

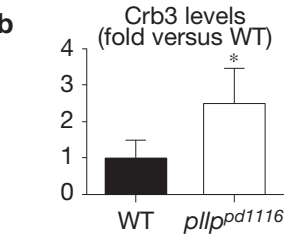

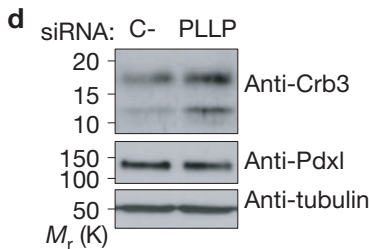

i AP Anti-GFP 90' chase
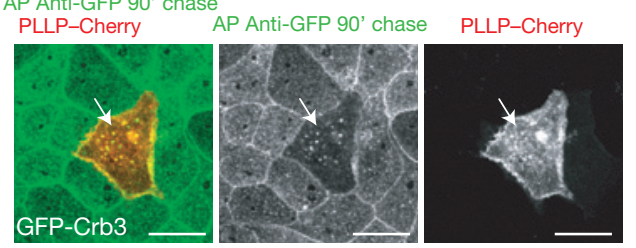

Figure 5 PLLP regulates Crb endocytosis. (a) Crb localization in WT and $p / l p^{p d 1116}$ mutant $144 \mathrm{hpf}$ larvae. Sections were stained with antipanCrb (red), phalloidin (green) and DAPI (blue). Arrows indicate Crb localization at apical membrane. Scale bars, $20 \mu \mathrm{m}$ (magnification, $10 \mu \mathrm{m}$ ). (b) Quantification of Crb levels in a. Data are represented as average foldincrease \pm s.d. (WT, $1.00 \pm 0.50$ fold; pllppd1116, $2.50 \pm 0.96$ fold; $n=6$ WT and 6 mutant fish from 3 independent experiments; ${ }^{*} P<0.005$ (Student's $t$-test)). (c) Localization of endogenous Crb3. MDCK cysts were fixed and labelled with anti-Crb3 (green), anti-ZO-1 (blue) and anti-E-cadherin (red). Arrowhead indicates apical Crb3. Arrows indicate suprajunctional Crb3. Scale bars, $5 \mu \mathrm{m}$. (d) Crb3 protein levels in PLLP-KD cysts. Data are expressed as mean \pm s.d. fold-increase versus control (control, $1 \pm 0.35$ fold; PLLP-KD, $2.31 \pm 0.13$ fold; $n=3$ extracts from 3 independent experiments, ${ }^{*} P<0.005$ (Student's $t$-test), statistics source data can be found in Supplementary Table 3). (e) Localization of endogenous Crb3 in PLLP-KD and Stx7-KD cysts. MDCK cells transfected with control, PLLP or Stx7-specific siRNAs were grown in cysts and labelled with anti-Crb3 (green), anti-ZO-1 (red) and anti-E-cadherin (blue). L, lumen. Arrowheads indicate apical Crb3. Arrows indicate suprajunctional Crb3. Scale bars, $5 \mu \mathrm{m}$. (f) Videomicroscopy of RUSH-Crb3a. RUSH-Crb3a MDCK cells were transfected with control or
PLLP siRNA and grown to form cysts. At $72 \mathrm{~h}$, biotin was added and cysts were recorded every minute until steady state. For endocytosis inhibition, cysts were treated with dynasore after biotin addition. Arrowheads indicate apical membrane. Arrows indicate tight junctions. Dashed lines mark the basal contour of the cysts. L, lumen. Scale bars, $5 \mu \mathrm{m}$. (g) Downmodulation of Crb3 in PLLP-GFP clones. PLLP-GFP-transfected cells were grown as monolayers for 4 days mixed with control MDCK cells, fixed and labelled with anti-Crb3 (red). The dashed line indicates the PLLP-GFP-expressing clone. Scale bars, $10 \mu \mathrm{m}$. (h) Quantification of $\mathbf{g}$. Medial and junctional Crb3 staining was measured as mean percentage of control fluorescence intensity \pm s.d. (GFP-neg, $100 \pm 8.3 \%$; PLLP-GFP, $73.6 \pm 7.7 \% ; n=20$ PLLP-GFP cells and 61 GFP-neg cells from 3 independent transfection experiments, ${ }^{*} P<0.01$ (Student's $t$-test)). (i) Pulse-chase endocytosis of GFP-Crb3a. MDCK cells stably expressing GFP-Crb3a were transfected with PLLP-Cherry. After $24 \mathrm{~h}$, the apical surface of the cells was incubated with anti-GFP to label $\mathrm{Crb} 3$ at $4{ }^{\circ} \mathrm{C}$, washed, and cells were returned to $37^{\circ} \mathrm{C}$ for $90 \mathrm{~min}$. Then, cells were fixed and stained with anti-rabbit-Alexa647 (green). Images are maximum $z$-stack projections. Arrows indicate endocytosed apical GFP-Crb3a. Scale bars, $10 \mu \mathrm{m}$. Uncropped images of blots/gels are shown in Supplementary Fig. 5. 


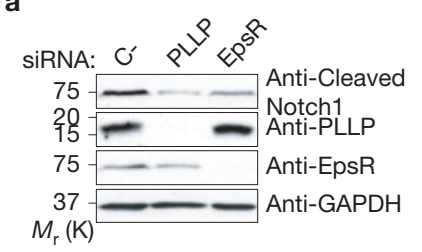

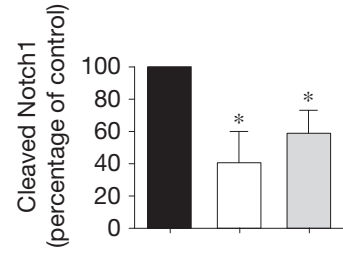

siRNA: C- PLLP EpsR b

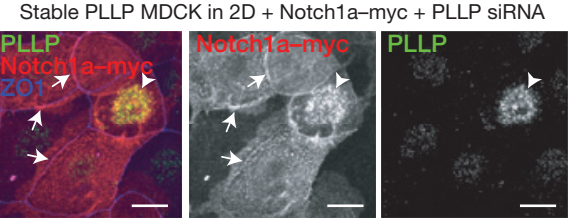

C

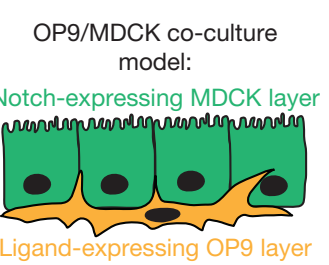

d OP9: Jagged-1 Delta-like 1

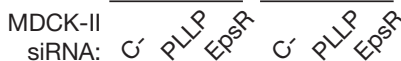

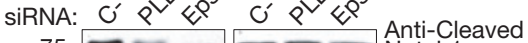

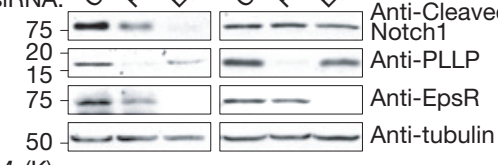

e oิ

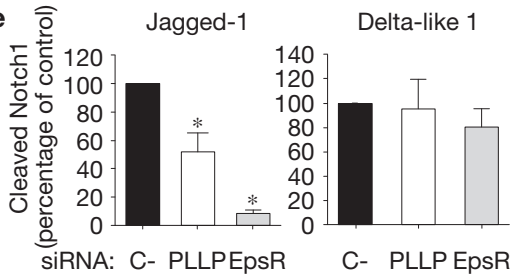

f

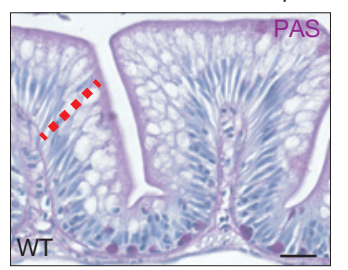

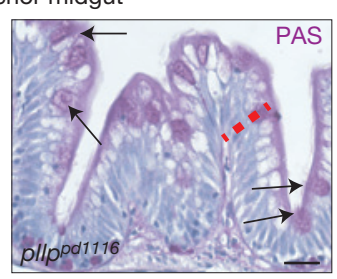

g
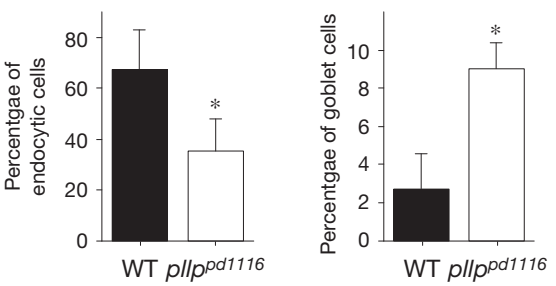

h
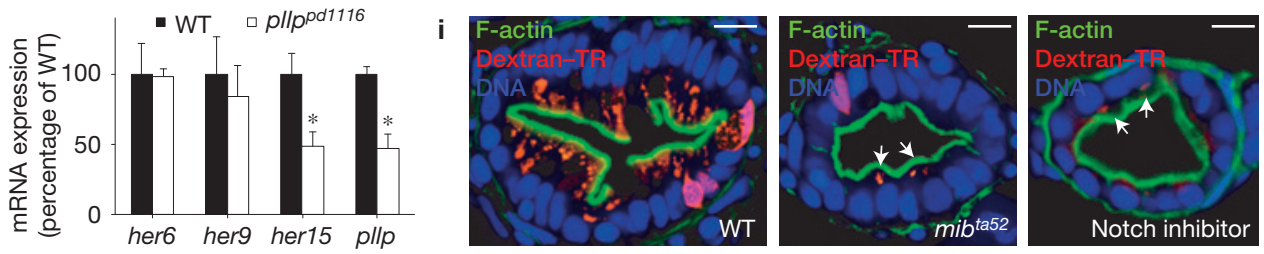

j

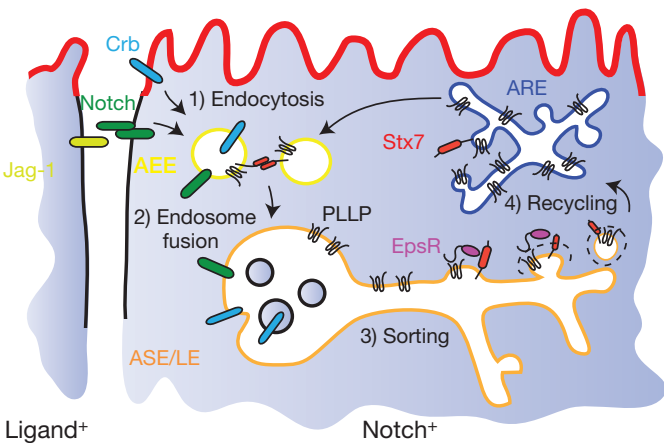

Mucosecretory compartment

Endocytic compartment WT
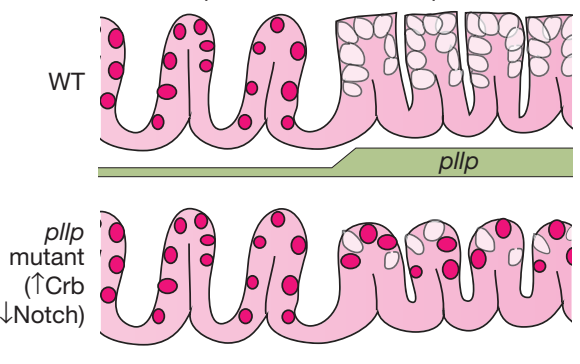

Figure 6 PLLP is required for Notch signalling. (a) Notch activation in PLLP-KD and EpsR-KD cysts. Cleaved Notch1 (NICD) protein levels were analysed by western blotting. Data are mean \pm s.d. as percentage of control (PLLP, $41 \pm 19 \%$, EpsR; $59 \pm 14 \%, n=3$ extracts from 3 independent experiments, ${ }^{*} P<0.05$ (Student's $t$-test), statistics source data can be found in Supplementary Table 3). (b) Notch localization in PLLP-KD cells. Cells were transfected with Notchla-myc and PLLP siRNA and labelled with anti-myc (red), anti-PLLP (green) and anti-ZO1 (blue). Arrows indicate junctional Notchla-myc in PLLP-depleted cells. Arrowheads indicate internal PLLP and Notch1a-myc co-localization in non-depleted cells. Scale bars, $10 \mu \mathrm{m}$. (c) MDCK-II/OP9 co-culture system for ligand-induced Notch1 transactivation assays. (d) Effect of PLLP-KD or EpsR-KD on ligand-specific Notch1 transactivation. MDCK cells stably expressing Notchla-myc were transfected with control, PLLP or EpsRspecific siRNAs and cultured with OP9 cells expressing Jagged-1 or Deltalike-1 and analysed by western blotting. (e) Quantification of Notch1 activity in d. Data are mean \pm s.d.\% of control cleaved Notch1 (PLLP-KD, $57.5 \pm 17.5 \%$; EpsR-KD, $8.2 \pm 5.6 \% ; n=3$ extracts from 3 independent experiments, ${ }^{*} P<0.05$ (Student's $t$-test), statistics source data can be found in Supplementary Table 3). (f) Intestinal morphology in adult p/lppd1116 posterior guts. Sections were stained with PAS (purple). Arrows indicate goblet cells. Red bars are placed to compare cell height. Scale bars, $20 \mu \mathrm{m}$. (g) Quantification of endocytic cells and goblet cells. Data are expressed as mean \pm s.d. percentage of total cells from 9 crypts per animal (endocytic cells: WT, $67.3 \pm 15.5 \%$; p/lp pd1116 $35.3 \pm 12.4 \%$; goblet cells: WT, $2.7 \pm 1.8 \%$; pllp pd1116 $9.0 \pm 1.3 \% ; n=6$ WT, 6 mutant fish from 2 independent experiments; ${ }^{*} P<0.005$ (Student's $t$-test)). (h) Expression of Notch-target hes-related genes in WT and p/lppd1116 adult guts. Data are mean \pm s.d. percentage of control expression ( $n=3$ extracts from 3 independent experiments; $* P<0.01$ (Student's $t$-test), statistics source data can be found in Supplementary Table 3). (i) Dextran endocytosis in Notch-inhibited larvae. DMSO (control) or $100 \mu \mathrm{M}$ DAPT (Notch inhibitor)treated larvae and $m i b 1^{\text {ta52b }}$ mutants were dextran-gavaged (red) and stained with phalloidin (green) and DNA (blue). Arrows indicate the few remaining cells that are able to endocytose dextran. Scale bars, $10 \mu \mathrm{m}$. (j) Model. Apical endosomal SNAREs, that is, Stx7, are polarized. In mature sorting endosomes PIlp recruits EpsR, which binds Stx7 to recycle it specifically to the apical pole through the ARE. The patterned expression of PIIp in the zebrafish intestine regulates $\mathrm{Crb}$ and Notch receptor endocytosis and results in functional patterning of the midgut by promoting terminal differentiation of absorptive endocytic cells. Uncropped images of blots/gels are shown in Supplementary Fig. 5. 
and BSA-conjugated $15 \mathrm{~nm}$ gold for ultrastructural analysis. IECs of pllp pd1116 larvae present alterations in apical endosome numbers and size distribution, and negligible levels of apical BSA-gold endocytosis compared with WT (Fig. 2d and Supplementary Fig. 1G, arrows, and Supplementary Fig. 1L). At later time points, juvenile pllp $p^{p d 1116}$ mutants (75\%) present disrupted intestinal folds and a 1.4-fold expansion in apical membrane size (Fig. 2e and Supplementary Fig. 1M), a phenotype resembling previous observations in Drosophila mutants with disrupted apical endocytosis ${ }^{5}$. The survival of pllp pd1116 mutants raised with a limited food supply was highly compromised compared with WT juveniles, suggesting that Pllp is necessary for efficient nutrient absorption (Supplementary Fig. $1 \mathrm{~N}$ ). We validated the specificity of phenotypes by crossing pllp $p^{p d 1116}$ mutants to TgBAC(pllpGFP) heterozygous animals. Pllp-GFP expression almost completely rescued both the endocytic and the cell-height phenotypes of the pllp pd1116 mutation, indicating that the lack of pllp expression in the mutants is the specific cause of the observed defects in IECs and that the fusion protein is functional (Supplementary Fig. 1O,P). To summarize, these results indicate that Pllp is required for apical endocytosis and epithelial morphogenesis in the gut and suggest a function in regulating terminal epithelial differentiation of posterior gut enterocytes.

\section{PLLP regulates formation of apical recycling endosomes}

The subapical localization of PLLP suggests its association with apical recycling endosomes (AREs), which are required for the recycling of endocytosed receptors back to the plasma membrane ${ }^{13}$. Endogenous Rab11, an ARE marker, partially co-localized with subapical Pllp (Fig. 2f, arrows, $r=0.64 \pm 0.09$ ). We observed that in pllp $p^{p d 1116}$ mutants Rab11 is mislocalized throughout the cytoplasm in posterior gut IECs, before any morphogenetic defects arise (Fig. 2g,h and Supplementary Fig. 1Q), suggesting that Pllp is required for the formation or maintenance of a polarized ARE compartment, and possibly for protein recycling at the onset of epithelial morphogenesis. In addition, electron micrographs of IECs revealed that $p l l p^{p d 1116}$ mutants presented a 2.6-fold decrease in the number of recycling/sorting tubules in apical endosomes compared with WT (Fig. 2d, insets, $2 \mathrm{i}$ and Supplementary Fig. 2A). In conclusion, Pllp is required for polarized Rab11 distribution in epithelial cells, suggesting that Pllp is required for the formation or maintenance of the ARE compartment, and possibly for protein recycling from apical sorting endosomes during epithelial morphogenesis.

\section{PLLP is required for epithelial morphogenesis and endosomal maturation in MDCK cysts}

To dissect more precisely the molecular function of PLLP we used the 3D-MDCK model system, which aptly recapitulates epithelial morphogenesis in vitro ${ }^{14}$. PLLP expression increases during lumen formation and localizes to the subapical compartment in 3D-MDCK cells (Fig. 3a,b). We also observed a similar pattern of expression in sections of mouse small intestine and kidney (Supplementary Fig. 2B,C), mimicking the subcellular localization and expression patterns observed in zebrafish. This common pattern of subcellular localization in epithelial tubes suggests a potential similar role in all of these tissues. Consistently, silencing of PLLP (PLLP-KD) results in morphogenetic defects (Fig. 3c-e and Supplementary Fig. 2D,E) and endolysosomal function defects (Fig. 2f,g). Furthermore, PLLP also partially co-localizes with Rab11 $(r=0.73 \pm 0.12)$ and PLLP-KD disrupts ARE polarization (Supplementary Fig. 2F,G). These results suggest a conserved role for PLLP in ARE polarization and endosomal maturation. Next, we tested whether PLLP overexpression is sufficient to enhance formation of Rab11 endosomes. Overexpression of PLLPGFP in monolayers of MDCK cells (2D) induces the formation of an enlarged Rab11-positive compartment, which co-localizes with early endosomal markers and induces the formation of acidic endosomes (Fig. 3h). This PLLP-GFP compartment consisted of clusters of vesicles that resembled ARE tubule vesicles (Supplementary Fig. $2 \mathrm{H}$ ). The TgBAC(pllp-GFP)pd1114 line, which overexpresses Pllp-GFP, presents a similar phenotype (Supplementary Fig. 2I). In summary, these experiments indicate that PLLP is required for endosomal maturation and ARE polarization, and furthermore that PLLP expression is sufficient to expand the ARE, and enhance formation of lytic acidic endosomes.

\section{PLLP interacts with EpsR to sort endosomal SNAREs to the recycling compartment}

To characterize the molecular mechanism associated with PLLP function, we devised an in vivo biotinylation assay (bioID) of PLLPproximal proteins (Fig. 4a). We uncovered 42 proteins likely to interact with PLLP in 3D-MDCK cells, including 20 proteins with trafficking functions and 9 SNARE proteins or SNARE regulators (Supplementary Tables 1 and 2). We identified Clint-1 (also known as Epsin-4 or EpsR, and hereafter termed EpsR) as the principal interacting partner of PLLP (Fig. 4b). EpsR belongs to the Epsin family of membrane-tubulating proteins and it is required for retrograde transport from late endosomes ${ }^{15-17}$. The amino-terminal ENTH domain of EpsR has been described to interact with several cargoes, including endosomal SNAREs, and is required for SNARE recycling ${ }^{18-20}$. We confirmed the interaction between endogenous PLLP and the carboxy-terminal domain of EpsR (Fig. 4c) and found that EpsR and PLLP partially co-localize in internal endosomes (Fig. 4d, arrows, and Supplementary Fig. 3A, arrows, $r=0.61 \pm 0.08$ ). PLLP-KD cells present a dispersed and decreased staining of both EpsR and Rab7 (Fig. 4e and Supplementary Fig. 3B) suggesting that PLLP is required for EpsR endosomal localization and maturation of degradative endosomes. EpsR silencing phenocopies PLLP-KD (Fig. 4f,l, and Supplementary Fig. 3C,D) and inhibits PLLP gain-offunction phenotypes (Supplementary Fig. 3E), indicating that EpsR binding to PLLP is required for the formation of apical Rab11-positive endosomes. Interestingly, the Drosophila EpsR homologue, Liquidfacets related $(\operatorname{lq} f R)$ is a regulator of epithelial cell morphology and regulates cell height in the follicle cells of the egg chamber ${ }^{21,22}$, which, together with our results, suggests that EpsR function in epithelial morphogenesis is conserved across bilateria.

Next, we investigated one of the canonical cargoes of EpsR, Syntaxin-7 (Stx7; ref. 18), which was also identified in our bioID assay (Supplementary Table 2). Stx7 is highly polarized to the subapical endosomal compartment in 3D-MDCK (Fig. 4g) and mouse intestine (Supplementary Fig. 3F). Furthermore, Stx7 and PLLP co-localize in the subapical compartment (Fig. 4g, arrowheads, $r=0.83 \pm 0.05$ ), and interact in these membrane domains as we observed using a probe-ligation assay ${ }^{23}$ (Supplementary Fig. 3G,H). Next, we addressed 
whether Stx7 subapical localization requires PLLP and EpsR. We found that silencing either PLLP or EpsR mislocalized Stx7 from the subapical compartment (Fig. 4g,h). FRAP (fluorescence recovery after photobleaching) analysis of subapical endosomes revealed similar recovery kinetics for both proteins ( $k_{\text {off }}$ Stx $7,0.022 \mathrm{~s}^{-1} ; k_{\text {off }}$ PLLP, $0.026 \mathrm{~s}^{-1}$ ), suggesting that they traffic in the same carriers to the subapical compartment (Fig. 4i,j, Supplementary Video 1). Moreover, we found that Stx7 silencing phenocopies PLLP and EpsR-KD (Fig. 4k,l and Supplementary Fig. 3I-L). Consistently, pllp ${ }^{\text {pd116 }}$ larvae showed a scattered distribution of Stx7, recapitulating PLLP-KD in MDCK cysts (Supplementary Fig. 3M).

Together these results indicate that endosomal SNAREs are polarized in the subapical compartment in epithelial cells and recycled back to the apical pole from sorting/late endosomes by interacting with PLLP and EpsR. These data also imply that formation of Rab11 endosomes depends on the maintenance of apical endosomal fusion and a cyclic dependence of both apical endocytosis and the recycling of the SNARE fusion machinery.

\section{PLLP regulates Crb endocytosis and Notch signalling during epithelial morphogenesis}

Our data suggest the possibility that PLLP levels could modulate endocytosis and degradation of apical protein receptors. Crb is a master regulator of epithelial morphogenesis and is regulated by endocytosis ${ }^{24-26}$. In vivo, pllp pd1116 mutants exhibited higher levels of Crumbs (Fig. 5a,b). In 3D-MDCK cells, Crumbs3 (Crb3) becomes progressively restricted to tight junctions (Fig. 5c and Supplementary Fig. 4A), correlating with the timing of PLLP induction, whereas PLLP-KD cells present Crb3 mislocalized to the apical plasma membrane and higher total levels of Crb3 (Fig. 5d,e, arrowheads, and Supplementary Fig. 4A-C). Crb3 mis-sorting could be explained by a defect in protein sorting to the tight junctions or by a defect in endocytosis at the apical plasma membrane. To dynamically address GFP-Crb3(a) localization we used the RUSH system ${ }^{27}$ (Supplementary Video 2). GFP-Crb3 is secreted first at the apical plasma membrane from where it then relocalizes to the tight junctions (Fig. 5f, top panels, arrows, and Supplementary Video 3). PLLP-KD or endocytosis inhibitor-treated 3D-MDCK cells fail to segregate Crb3 later to the tight junctions (Fig. 5f, middle and bottom panels, Supplementary Videos 4 and 5). Moreover, PLLP overexpression is sufficient to induce Crb3 endocytosis and downmodulation (Fig. 5g-i). Our results suggest that PLLP expression is necessary and sufficient to control Crb3 levels directly through regulation of apical endocytosis.

Notch signalling is required for absorptive intestinal cell differentiation across evolution ${ }^{25,28,29}$ and the Stx7 homologue, avl, is required for Notch signalling in Drosophila ${ }^{30}$. PLLP-KD or EpsR-KD reduces activated Notch (NICD) levels by 60 and $40 \%$ respectively (Fig. 6a) and PLLP-KD inhibits full-length Notchla localization in endosomes (Fig. 6b, arrows), whereas the overexpression of PLLPGFP is sufficient to induce Notch1a internalization. These results suggested that PLLP is required for Notch-receptor endocytosis and activation. However, Notch activation also requires Epsin-mediated endocytosis of Notch ligands ${ }^{31,32}$. To further analyse whether PLLP and EpsR are required for receptor or ligand activation, we cultured Notchla-expressing MDCK cells over mesenchymal OP9 cell layers stably expressing Jagged-1 (also known as Serrate) or Delta-like 1, the main ligands expressed in the zebrafish gut ${ }^{33}$. PLLP-KD and EpsR-KD inhibited Notch 1 transactivation specifically by Jagged-1, and not by Delta-like1 (Fig. 6c-e and Supplementary Fig. 4D). Then, we cocultured Notch1-expressing MDCK cells with Jagged-1-MDCK cells, and confirmed that Notch-receptor cells require the expression of both PLLP and EpsR, whereas EpsR expression seems to be also required in the ligand-presenting cells (Supplementary Fig. 4E-H). These results indicate that PLLP is induced in Notch-receptor cells to specifically regulate Notch activity in these cells.

Next, we analysed Notch signalling in vivo. The pllp pd1116 mutants present a reduced number of terminally differentiated vacuolated cells and a threefold increase of PAS-positive mucosecretory cells (Fig. 6f,g). Moreover, pllp pd1116 mutants show a marked decrease in expression of the bona fide Notch-effector gene her15 (Fig. 6h), similarly to what has been previously reported for Mindbomb (mib) mutations, in which Jagged-family ligands are unable to signal to Notch-positive cells ${ }^{33}$. Together, these results indicate that Pllp is required for Notch signalling, terminal differentiation of posterior gut enterocytes and inhibition of secretory cell differentiation in the posterior midgut. To analyse whether Notch signalling is required for terminal differentiation of the posterior vacuolated IECs, we gavaged $144 \mathrm{hpf}$ Mindbomb mib $1^{\text {ta52b }}$ mutant larvae and Notch inhibitor-treated larvae. Both exhibited a significant reduction in the number of endocytic cells and the size of endosomes (Fig. 6i, arrows). Together, these experiments indicate that Pllp controls Notch activity, which is essential for absorptive enterocyte terminal differentiation in the posterior midgut.

\section{DISCUSSION}

Here, we characterized a developmentally regulated mechanism to induce apical endocytosis through the regulation of SNARE sorting that is necessary for epithelial morphogenesis (Fig. 6j). We propose that endosomal SNAREs are polarized to different endosomal networks at the apical and basolateral domains. Apical SNARE sorting is controlled to regulate the rate of apical protein endocytosis, involved in receptor degradation and signalling. We describe that expression of a previously uncharacterized protein, PLLP, induces SNARE recycling through its interaction with the membrane-tubulating clathrin adaptor EpsR (Fig. 6j).

In addition, our experiments demonstrate that PLLP is induced in a time- and space-specific manner to regulate the in vivo differentiation of a notch-mediated highly endocytic absorptive cell population in the zebrafish midgut (Fig. 6j). Epithelial morphogenesis is a finely regulated process in which epithelial cells conduct a delicate balancing act between differentiation and proliferation that becomes deregulated in different types of human carcinoma ${ }^{34}$. Epithelial cell differentiation greatly depends on the establishment of cellular junctions and polarity complexes that serve to organize the physiology of mature epithelial tissues. These polarity complexes, such as the Crb complex, crosstalk with proliferation pathways, such as the Notch pathway, to prevent overgrowth and, at the same time, to provide a functional population of highly differentiated epithelial cells ${ }^{4}$. Our experiments indicate that PLLP fine-tunes Notch signalling for differentiation of posterior gut absorptive cells. Adult pllp mutants presented posterior guts that resembled more anterior compartments, with a reduced population of vacuolated cells and increased populations of mucosecretory cells (Fig. 6j). 
Pllp is also expressed in several other epithelial-like cell types in zebrafish not described here, such as a subpopulation of skin cells, the sheath cells of the notochord, and the neuromasts of the lateral line. Interestingly, asymmetric proliferation and differentiation of these cell types also depend on Notch signalling ${ }^{35-37}$. Further studies will be directed to understanding the role of PLLP in fine-tuning Notch activity during development of these organs.

\section{METHODS}

Methods and any associated references are available in the online version of the paper.

Note: Supplementary Information is available in the online version of the paper

\section{ACKNOWLEDGEMENTS}

We thank C. M. Ruiz-Jarabo for her comments on the manuscript and members of the Martin-Belmonte laboratory and Bagnat laboratories for helpful discussions. We thank A. Alvers (Duke University, North Carolina, USA) for helping in the isolation of gut cells, J. Cocchiaro and L. Marjoram (Duke University, North Carolina, USA) for help in gavaging experiments, B. Margolis (University of Michigan, Michigan, USA) for the Crb3/panCrb antibody, R. Jahn (Max Planck Institute for Biophysical Chemistry, Germany) for Stx7 plasmids, M. Robinson (University of Cambridge, UK) for EpsR plasmids, and R. Kopan and J. L. de la Pompa (CNIC, Spain) for Notch plasmids and mib1 $1^{\text {tas2b }}$ embryos. We also thank M. Guerra at the EM Unit for skillful technical assistance. This work was supported by grants from the MINECO (BFU2011-22622) and CONSOLIDER (CSD2009-00016) to F.M-B, by grant SAF2013-44857-R to M.L.T. by NIH innovator grant 1DP2OD006486 to M.B., and by grant AGL2013-48998-C2-2-R to G.A. A.E.R-F was supported by a CSIC JAE PhD fellowship. M.B-F. is a recipient of a Fundación Obra Social 'La Caixa' PhD fellowship. G.A. was supported by the Amarouto Program for senior researchers from the Comunidad Autónoma de Madrid.

\section{AUTHOR CONTRIBUTIONS}

A.E.R-F., M.B. and F.M-B. designed the experiments; A.E.R-F., J.B., M.B-F. and G.A. carried out the experiments; A.E.R-F. and F.M-B. wrote the manuscript; G.B. and F.P. designed and constructed RUSH experimental tools; M.J.G-L. and M.L.T. designed and constructed the Notch-ligand tools; N.R-R., M.A.A. and J.M. produced and characterized the mammalian PLLP antibody; A.E.R-F. and G.A. designed and carried out the electron microscopy experiments.

\section{COMPETING FINANCIAL INTERESTS}

The authors declare no competing financial interests.

Published online at www.nature.com/doifinder/10.1038/ncb3106

Reprints and permissions information is available online at www.nature.com/reprints

1. Rodriguez-Boulan, E. \& Macara, I. G. Organization and execution of the epithelial polarity programme. Nat. Rev. Mol. Cell Biol. 15, 225-242 (2014).

2. Eaton, S. \& Martin-Belmonte, F. Cargo sorting in the endocytic pathway: a key regulator of cell polarity and tissue dynamics. Cold Spring Harb. Perspect. Biol. 6 (2014).

3. Fabrowski, P. et al. Tubular endocytosis drives remodelling of the apical surface during epithelial morphogenesis in Drosophila. Nat. Commun. 4, 2244 (2013).

4. Richardson, E. C. \& Pichaud, F. Crumbs is required to achieve proper organ size control during Drosophila head development. Development 137, 641-650 (2010).

5. Lu, H. \& Bilder, D. Endocytic control of epithelial polarity and proliferation in Drosophila. Nat. Cell Biol. 7, 1232-1239 (2005).

6. Bokel, C. \& Brand, M. Endocytosis and signaling during development. Cold Spring Harb. Perspect. Biol. 6 (2014).

7. Bagnat, M., Cheung, I. D., Mostov, K. E. \& Stainier, D. Y. Genetic control of single lumen formation in the zebrafish gut. Nat. Cell Biol. 9, 954-960 (2007).

8. Alvers, A. L., Ryan, S., Scherz, P. J., Huisken, J. \& Bagnat, M. Single continuous lumen formation in the zebrafish gut is mediated by smoothened-dependent tissue remodeling. Development 141, 1110-1119 (2014).
9. Sanchez-Pulido, L., Martin-Belmonte, F., Valencia, A. \& Alonso, M. A. MARVEL: a conserved domain involved in membrane apposition events. Trends Biochem. Sci. 27, 599-601 (2002).

10. Navis, A., Marjoram, L. \& Bagnat, M. Cftr controls lumen expansion and function of Kupffer's vesicle in zebrafish. Development 140, 1703-1712 (2013).

11. Cocchiaro, J. L. \& Rawls, J. F. Microgavage of zebrafish larvae. J. Visualized Experiments: JoVE e4434 (2013).

12. Cermak, T. et al. Efficient design and assembly of custom TALEN and other TAL effector-based constructs for DNA targeting. Nucleic Acids Res. 39, e82 (2011).

13. Golachowska, M. R., Hoekstra, D. \& van, I. S. C. Recycling endosomes in apical plasma membrane domain formation and epithelial cell polarity. Trends Cell Biol. 20, 618-626 (2010).

14. Galvez-Santisteban, M. et al. Synaptotagmin-like proteins control the formation of a single apical membrane domain in epithelial cells. Nat. Cell Biol. 14 838-849 (2012).

15. Saint-Pol, A. et al. Clathrin adaptor epsinR is required for retrograde sorting on early endosomal membranes. Dev. Cell 6, 525-538 (2004).

16. Mills, I. G. et al. EpsinR: an AP1/clathrin interacting protein involved in vesicle trafficking. J. Cell Biol. 160, 213-222 (2003).

17. Hirst, J., Motley, A., Harasaki, K., Peak Chew, S. Y. \& Robinson, M. S. EpsinR: an ENTH domain-containing protein that interacts with AP-1. Mol. Biol. Cell 14, 625-641 (2003).

18. Miller, S. E., Collins, B. M., McCoy, A. J., Robinson, M. S. \& Owen, D. J. A SNAREadaptor interaction is a new mode of cargo recognition in clathrin-coated vesicles. Nature 450, 570-574 (2007).

19. Chidambaram, S., Zimmermann, J. \& von Mollard, G. F. ENTH domain proteins are cargo adaptors for multiple SNARE proteins at the TGN endosome. J. Cell Sci. 121, 329-338 (2008)

20. Chidambaram, S., Mullers, N., Wiederhold, K., Haucke, V. \& von Mollard, G. F. Specific interaction between SNAREs and epsin N-terminal homology (ENTH) domains of epsin-related proteins in trans-Golgi network to endosome transport. J. Biol. Chem. 279, 4175-4179 (2004).

21. Leventis, P. A. et al. Liquid facets-related (IqfR) is required for egg chamber morphogenesis during Drosophila oogenesis. PIOS ONE 6, e25466 (2011).

22. Lee, J. H., Overstreet, E., Fitch, E., Fleenor, S. \& Fischer, J. A. Drosophila liquid facets-Related encodes Golgi epsin and is an essential gene required for cell proliferation, growth and patterning. Dev. Biol. 331, 1-13 (2009).

23. Leuchowius, K. J., Weibrecht, I., Soderberg, O. in Current Protocols in Cytometry (eds Robinson, J. P. et al.) Ch. 9 (Wiley, 2011).

24. Thompson, B. J., Pichaud, F. \& Roper, K. Sticking together the Crumbs-an unexpected function for an old friend. Nat. Rev. Mol. Cell Biol. 14, 307-314 (2013).

25. Fre, S., Bardin, A., Robine, S. \& Louvard, D. Notch signaling in intestinal homeostasis across species: the cases of Drosophila, Zebrafish and the mouse. Exp. Cell Res. 317, 2740-2747 (2011)

26. Harder, J. L., Whiteman, E. L., Pieczynski, J. N., Liu, C. J. \& Margolis, B. Snail destabilizes cell surface Crumbs3a. Traffic 13, 1170-1185 (2012).

27. Boncompain, G. et al. Synchronization of secretory protein traffic in populations of cells. Nat. Methods 9, 493-498 (2012).

28. VanDussen, K. L. et al. Notch signaling modulates proliferation and differentiation of intestinal crypt base columnar stem cells. Development 139, 488-497 (2012).

29. Van Es, J. H. et al. Notch/gamma-secretase inhibition turns proliferative cells in intestinal crypts and adenomas into goblet cells. Nature 435, 959-963 (2005).

30. Vaccari, T., Lu, H., Kanwar, R., Fortini, M. E. \& Bilder, D. Endosomal entry regulates Notch receptor activation in Drosophila melanogaster. J. Cell Biol. 180, 755-762 (2008)

31. Wang, W. \& Struhl, G. Distinct roles for Mind bomb, Neuralized and Epsin in mediating DSL endocytosis and signaling in Drosophila. Development 132 , 2883-2894 (2005).

32. Wang, W. \& Struhl, G. Drosophila Epsin mediates a select endocytic pathway that DSL ligands must enter to activate Notch. Development 131, 5367-5380 (2004).

33. Crosnier, C. et al. Delta-Notch signalling controls commitment to a secretory fate in the zebrafish intestine. Development 132, 1093-1104 (2005).

34. Martin-Belmonte, F. \& Perez-Moreno, M. Epithelial cell polarity, stem cells and cancer. Nat. Rev. Cancer 12, 23-38 (2012).

35. Yamamoto, M. et al. Mib-Jag1-Notch signalling regulates patterning and structural roles of the notochord by controlling cell-fate decisions. Development 137 , 2527-2537 (2010).

36. Wibowo, I., Pinto-Teixeira, F., Satou, C., Higashijima, S. \& Lopez-Schier, H. Compartmentalized Notch signaling sustains epithelial mirror symmetry. Development 138, 1143-1152 (2011).

37. Liu, Y., Pathak, N., Kramer-Zucker, A. \& Drummond, I. A. Notch signaling controls the differentiation of transporting epithelia and multiciliated cells in the zebrafish pronephros. Development 134, 1111-1122 (2007). 


\section{METHODS}

Plasmids. Rat Stx7-GFP plasmids were kind gifts from R. Jahn (Max Planck Institute for Biophysical Chemistry, Germany). Human Rablla-GFP, canine PLLP-GFP and canine PLLP-Cherry were constructed by PCR and cloned into $\mathrm{pEGFP/mCherry} \mathrm{vectors} \mathrm{(Clontech).} \mathrm{The} \mathrm{siRNA} \mathrm{no.} \mathrm{2-resistant}(\mathrm{R})$ variants were generated by introducing synonymous mutations with the Quikchange XLII kit (Stratagene). Canine PLLP-myc/myc-birA* was constructed by PCR and cloned into pCR3.1(+) (Invitrogen). Human EpsR-GFP, GST-N-EpsR and GST-C-EpsR were gifts from S. Robinson (University of Cambridge, UK). Rab7-GFP was from R. Puertollano (NIH, USA). The BAC clones containing pllp and lamp2 genes were obtained from Source Biosciences ( $p l l p$ HUKGB735N1073Q/DKEY73N10 and lamp2 HUKGB735N0515Q/DKEY-15N5). GFP-Crb3a were kindly provided by D. Bryant (Beatson Institute, University of Glasgow, UK). The spacerGFP/RFP sequence was cloned by BAC homologous recombination in bacteria as previously reported ${ }^{10}$. Full-length Notchla-myc was obtained from Addgene (plasmid 41728).

Antibodies and reagents. The polyclonal antibody (pAb) against mammalian PLLP (1:500 immunofluorescence (IF) on cold methanol/acetone fixation, 1:1,000 western blotting $(\mathrm{WB})$ ) was designed and generated in rabbits by injecting a combination of cytoplasmic peptides from the human PLLP sequence as previously described ${ }^{38}$. Podocalyxin/gp135 (1:500 IF, 1:1,000 WB) was a gift from G. Ojakian (State University of New York Downstate Medical Center, USA). Crb3 pAb (1:250 IF on acetone fixation, 1:1,000 WB) was a gift from B. Margolis (University of Michigan, Ann Arbor, USA). Cleaved Notch1 rabbit mAb (no. 4147, 1:1,000 WB, Cell Signaling), GAPDH mAb 6C5 (no. sc-32233, 1:1,000 WB, Santa Cruz Biotechnologies), ßcatenin pAb (no. sc-7199, 1:500 IF, Santa Cruz Biotechnologies), E-cadherin mAb (rr1, 1:500 IF, DSHB), GFP mAb (no. 11814460001, 1:100 IF, 1:1,000 WB, Roche), GFP pAb (no. A-11122, 1:1,000 immunoprecipitation (IP), 1:1,000 IF, 1:2,000 WB, Life Technologies), myc 9E10 $\mathrm{mAb}$ (no. 11667149001, 1:1,000 IF on acetone fixation, Roche), EpsR mAb (no. 86046, 1:100 IF on methanol/acetone fixation, 1:500 WB, Abcam), Stx7 pAb (no. 110072, 1:100 on IF on acetone fixation, 1:500 WB, Synaptic Systems), Rab11 pAb (no. 715300, 1:500 IF, 1:500 WB, Life Technologies), tubulin (no. T9026, DM1A, 1:1,000 WB, Sigma-Aldrich) and EEA1 mAb (no. 610457, 1:500 IF, BD Biosciences) were used as primary antibodies. Peroxidase-conjugated antibodies were used for western blotting (Jackson Immunoresearch). Alexa405/488/555/647conjugated phalloidin or secondary antibodies were used for immunofluorescence. DAPI, ToPRO3, Lysotracker-red and dextran-Texas red (TR) were from Life Technologies. Dynasore (MERCK) was used at $100 \mu \mathrm{M}$ in culture medium to inhibit dynamin, and DAPT (Sigma-Aldrich) at $100 \mu \mathrm{M}$ was used to inhibit Notch cleavage.

Transgenic animals and mutants. Zebrafish stocks were maintained at $28^{\circ} \mathrm{C}$. The zebrafish lines used were EK, TgBAC(cldn15la-GFP)pd1034 (ref. 8), TgBAC(pllpGFP)pd1114, TgBAC(pllp-GFP)pd1115, TgBAC(lamp2-spRFP)pd1117, mib1 $1^{\text {tas2b }}$ (ref. 39) and $p l l p^{\text {pd1116 }}$. Zebrafish BAC lines were generated as previously described ${ }^{10}$. Zebrafish that were found dead, not swimming or without heartbeat were excluded from the analyses. To randomize animal selection, we followed common protocols for unbiased tank fishing. Genotypes were determined by fin clipping. Larvae and juveniles were pipetted into fixation media without preselecting them on the low-magnification scope. Except where noted, larvae were $144 \mathrm{hpf}$, juveniles were $16 \mathrm{dpf}$ and adults were 4 months old. Experiments were supervised by the bioethics committee of the Centro de Biología Molecular 'Severo Ochoa' (CSIC) and performed in compliance with bioethical regulations of the European Commission. No statistical method was used to predetermine sample size for treatment groups. There was no requirement for animal randomization during the course of the animal studies.

TALEN-mediated editing. Three TALENs were designed to target the first exon of pllp using TALEN targeter and constructed using Golden Gate assembly into the pCS2-TAL3DD/RR vectors using the Addgene v2.0 $\mathrm{kit}^{12,40}$. The TALEN used to generate the pllp pd1116 mutant allele reported here was designed to target the following sequence of Danio rerio pllp exon1: 5'-TTGACATGGGTTTTATcaagagcattcctggaa TACTGCTTATAGCCGA- $3^{\prime}$, and composed of the following TAL effector domains: pCS2-TAL3DD_pllpE1 NG NN NI HD NI NG NN NN NN NG NG NG NG NI NG; pCS2-TAL3RR_pllpE1 HD NN NN HD NG NI NG NI NI NN HD NI NN NG NI. Zebrafish were injected into the yolk at the one-cell stage with $200 \mathrm{pg}$ total TALEN RNA and $100 \mathrm{pg}$ of dsRed RNA to select correctly injected embryos. Mutant alleles were identified by defective BsmI digestion of the PCR product generated with the following primers: FW: 5'-CTGGGAAGGTCAGCACTCAG-3'; RV: 5'-AC GGAACAGAAAAGTGGGTGT-3'. The BsmI-undigested PCR band was T/A cloned into the pGEM-T vector for allele sequencing. The experiments shown here were performed on F4/F5 fish and larvae.
Fish gavaging. Zebrafish larvae from $144 \mathrm{hpf}$ were tricained for $5 \mathrm{~min}$ and immersed in $3 \%$ methylcellulose. Microforged capillary needles were used to microinject $10 \mathrm{nl}$ of a 1:4:1 dextran-TR/water/Phenol-red solution. Methylcellulose was washed off and fish were incubated at $28^{\circ} \mathrm{C}$ for $2 \mathrm{~h}$ before confocal microscopy analysis or fixation.

Endocytosis assay in cells. MDCK cells stably expressing GFP-Crb3a were cultured as monolayers, washed with cold $1 \%$ FBS-supplemented MEM and placed on ice for $15 \mathrm{~min}$. Then, coverslips were placed on a $100 \mu \mathrm{l}$ drop of cold $1 \%$ FBS MEM containing a 1:10,000 dilution of the polyclonal GFP antibody at $4{ }^{\circ} \mathrm{C}$ for $30 \mathrm{~min}$. Coverslips were washed 3 times with 1\% FBS-supplemented MEM and placed on plates containing warm MEM and cultured at $37^{\circ}$ for $90 \mathrm{~min}$. Cells were washed in $\mathrm{Ca} / \mathrm{Mg}-\mathrm{PBS}$, fixed and stained for immunofluorescence.

In situ hybridization. The probe to detect the pllp transcript by in situ hybridization was PCR amplified from 5 dpf larval cDNA and ligated into pGEMTEasy (Promega). In situ hybridization was performed as previously described. The plasmids were linearized and digoxygenin-labelled RNA was generated using the DIG-labelled nucleotides (Roche) and T7 polymerase (NEB). Stained embryos were imaged on a Discovery.V20 stereoscope (Zeiss) with an Achromat S $1.0 \times$ lens.

Fish sectioning and analysis. Zebrafish embryos and larvae from different time points were fixed overnight in PBS-buffered 4\% PFA (Sigma), washed twice in PBS and embedded in PBS-buffered 4\% low-melt agarose blocks. Blocks were cut in $200 \mu \mathrm{m}$ sections using a Vibratome (Leica). Sections were blocked/permeabilized with PBS-3\%BSA containing 0.5\% Tx100 and then incubated with the indicated antibodies. Stained sections were mounted using DAPI-Fluoromount or DAPIVectashield. Sectioned fish were analysed on a confocal microscope. Adult fish were paraffin embedded, sectioned in $5 \mu \mathrm{m}$ slices, dewaxed and stained with haematoxylin/eosin (HE) or periodic acid-Schiff (PAS). Data were repeated using three different sections from the same tissue and/or genotype. Representative images are shown.

Cell culture and stable cell lines. MDCK type 2 (MDCK-II, MDCK.2) cells were obtained from ATCC and grown as described previously ${ }^{41}$. MDCK cells stably expressing PLLP-GFP, PLLP-Cherry, GFP-Rab7, GFP-VAMP8, GFP-Stx7 and EpsR-GFP were made by transfection using Lipofectamine 2000 (Life Technologies) and clones were selected by treating cells with G418 $\left(0.5 \mathrm{mg} \mathrm{ml}^{-1}\right)$. The Notchlamyc stable cell line was made by co-transfection with the blasticidin-resistant gene (pBlast) and selection for $10 \mathrm{~d}$ with $0.5 \mu \mathrm{g} \mathrm{ml}^{-1}$ blasticidin. Mycoplasma testing was regularly performed. To prepare cysts in Matrigel, cells were trypsinized to a singlecell suspension of $2 \times 10^{4}$ cells $\mathrm{ml}^{-1}$ in $2 \%$ Matrigel and plated in coverglass bottom chambers (IBIDI) covered with Matrigel. Cysts were grown and fixed at indicated time points.

Confocal microscopy and videomicroscopy. Immunofluorescence of cysts was previously described ${ }^{41}$. Fixed cells in 3D cultures were analysed mounted in ProLong Gold antifade reagent. Fixed cells in monolayers were analysed mounted in Fluoromount. Cysts were analysed on a 510 or 710 LSM confocal microscope (Carl Zeiss) using a $\times 63$ NA 1.4 oil Plan-Apochromat objective and a $\times 63$ NA 1.2 water C-Apochromat Corr (for live-cell and cyst imaging) and ZEN software suite (Carl Zeiss). Fish sections and whole-mounts were analysed on a SP5 confocal microscope (Leica) with a $\times 10 / 0.40 \mathrm{HC}$ PL APO air objective, a $\times 20 / 0.70$ HC PL APO oil objective, and a $\times 40 / 1.25-0.75$ HCX PL APO oil objective, using Application Suite software (Leica). For image processing, we used FIJ/ImageJ (National Institutes of Health). For videomicroscopy and 3D reconstitutions, we processed maximum $z$-projections of all stacks using ImageJ software. For quantification of lumen formation, MDCK cysts with a single actin/Podxl staining at the interior surface and $\beta$-catenin facing the ECM were identified as normal lumina. We excluded cyst formation experiments that presented lower than 50\% normal lumen formation (at $48 \mathrm{~h}$ ) or $60 \%$ (at $72 \mathrm{~h}$ ). Cysts at $72 \mathrm{~h}$ presenting two large lumina were considered 'normal.' To randomize cyst or cell counting, we randomly selected fields using low magnification, and then counted or took images at higher magnification for measurements. Immunofluorescence experiments in cell lines were performed three independent times and images shown are representative from samples that were used for quantification. For fluorescence intensity quantification in MDCK cysts, background was removed, maximum projections of optimal volumetric slicing of single cells were obtained and fluorescence signal was quantified as total integrated density per cell. For GFPStx7 or Rab11 signal polarization quantification, perpendicular linear intensity profiles were measured. For Crb measurements in zebrafish, apical membrane regions $(1 \mu \mathrm{m} \times 3 \mu \mathrm{m}$ rectangle) were chosen and measured from 10 cells per fish section. 
RUSH assay. The RUSH protocol was performed as previously described, with the following modifications. MDCK cysts stably expressing GFP-SBP-Crb3a and streptavidin-KDEL were grown in Mattek coverglass bottom plates for $72 \mathrm{~h}$, washed and incubated with $10 \mathrm{mM}$ Hepes-buffer, $1 \%$ serum-supplemented phenol-redfree MEM and imaged using a 510 LSM confocal microscope (Zeiss). Biotinsupplemented MEM $(40 \mu \mathrm{M})$ was added at $t=0$ when image acquisition started. For endocytosis inhibitor experiments, cysts were treated with $100 \mu \mathrm{M}$ dynasore at $40 \mathrm{~min}$ after biotin addition, when most Crb3 protein had reached the apical surface.

Electron microscopy. For BSA-gold endocytosis TEM, $144 \mathrm{hpf}$ larvae were gavaged with $15 \mathrm{~nm}$ gold-conjugated BSA (EM Laboratory, Utrecht University) supplemented with dextran-TR for $2 \mathrm{~h}$. Then, larvae were fixed in $2 \%$ (w/vol) PFA, $2 \%$ (w/vol) glutaraldehyde in $0.1 \mathrm{M}$ phosphate buffer $(\mathrm{PB}$, at $\mathrm{pH} 7.4)$ for $2 \mathrm{~h}$ at room temperature and overnight at $4{ }^{\circ} \mathrm{C}$. Subsequently, posterior midgut sections were embedded in Epon resin, sectioned using a ultramicrotome (Ultracut E, Leica), and stained with uranyl acetate and lead citrate and imaged at $80 \mathrm{kV}$ using a JEM1010 Jeol microscope. For immunogold electron microscopy, $144 \mathrm{hpf}$ zebrafish larvae were fixed in $2 \%$ (w/vol) PFA and $0.2 \%$ (w/vol) glutaraldehyde in $0.1 \mathrm{M}$ phosphate buffer $(\mathrm{PB}$, at $\mathrm{pH} 7.4)$ for $2 \mathrm{~h}$ at room temperature and kept in $1 \%(\mathrm{w} / \mathrm{vol}) \mathrm{PFA}$ in $\mathrm{PB}$ at $4{ }^{\circ} \mathrm{C}$. Subsequently, posterior gut sections were embedded in $10 \%(\mathrm{w} / \mathrm{vol})$ gelatine, and processed for cryosectioning. Guts were sectioned along the apicobasal axis on an EM FCS cryo-ultramicrotome (Ultracut UCT, Leica) at $-120^{\circ} \mathrm{C}$. For immunogold labelling, thawed 75-nm-thick cryosections were incubated with rabbit anti-GFP (1:500, Life Technologies) followed by protein A conjugated to 15-nm gold particles (EM Laboratory, Utrecht University). Sections were stained with a mix of $1.8 \%$ methylcellulose and $0.4 \%$ uranyl acetate and imaged at $80 \mathrm{kV}$ using a JEM1010 Jeol microscope and a $4 \times 4 \mathrm{k}$ CMOS F416 camera from TVIPS (Gauting).

RNAi and western blot. Twenty-five nucleotide stealth siRNA duplexes targeting mRNA sequences of canine PLLP and Stx7 were purchased from Life Technologies. Twenty-five nucleotide siRNA duplexes targeting EpsR were purchased from SigmaAldrich using dTdT overhangs. Sequences were submitted to BLAST search to ensure targeting specificity and minimize off-targets. MDCK cells were transfected using AMAXA Nucleofector-II equipment, reagents and protocols (Lonza). Cells were transfected with $10 \mu \mathrm{l}$ of siRNA $(200 \mu \mathrm{M})$, plated in 6 -well plates, cultured for $24 \mathrm{~h}$, trypsinized and then plated to grow cysts for the indicated time points. Total cell lysates were analysed by western blotting. Immunoblots shown are representative of experiments that were repeated and reproduced at least three independent times. For some challenging experiments and antibodies, the representative blots are ones that show the least nonspecific background and have a low signal-to-noise ratio.

The siRNAs targeted the following sequences: control: 5'-CCUUCGGGUGGAA CAUGCUCUCUUU-3' PLLP no. 1: 5'-CUGCUGCAGCUGGUGCUGGGGCUG C-3' PLLP no. 2: 5' -CCUCUGGCUGGUGACAAUCGUCUUU-3' PLLP no. 3: 5'-C CUAAGGAAUCGGGAUCCUUCCUCU-3' EpsR no. 1: 5' -CCUAUGAAUGUGA UGACCCAAAGUU-3', EpsR no. 2: 5' -CAUGAACAUAGGGAUGUCAACUGCU3', EpsR no. 3: 5' -AAGGAGCAGAUUGAAUGAAGGAUUU-3', Stx7 no. 1: 5' -UUC AGGUGAAUCUUGAGGUGUUCCA-3' Stx7 no. 2: 5'-CAGAAGAUGACCUCC GCCUUAUUCA-3' Stx7 no. 3: 5' -UAGAGAAUGUAGUGCAAUAGUGUGC-3'.

Probe ligation assay. The probe ligation assay (O-LINK) was performed using anti-PLLP (rabbit polyclonal) and anti-GFP (mouse, Roche) according to the manufacturer's instructions.

In vivo biotinylation of PLLP-proximal proteins. MDCK cells stably expressing the promiscuous mutant $(\mathrm{R} 118 \mathrm{G})$ of the humanized bacterial biotin-ligase (birA*) or canine PLLP-myc/myc-birA* constructs were incubated with $50 \mu \mathrm{M}$ biotin for $16 \mathrm{~h}$ and lysed using $4 \%$ SDS, and biotinylated peptides were purified using streptavidin-coated magnetic beads (Genscript). The bioID technique was performed as previously described ${ }^{42}$ and eluted peptides were analysed by liquid chromatography tandem mass spectrometry and peptide-mass fingerprinting, considering up to 2 biotinylations per peptide, in collaboration with the Proteomics unit at Centro Nacional de Biotecnología (CSIC).

Pulldowns. The full-length EpsR construct is extremely protease sensitive, so the NH2-terminal ENTH domain (amino acids 1-165) and the COOHterminal domain (amino acids 165-625) of human EpsR were expressed separately as previously published ${ }^{17}$. GST-fusion constructs were transformed into BL21 Escherichia coli and expressed by incubating bacterial clones at $30^{\circ} \mathrm{C}$ using $0.5 \mathrm{mM}$ IPTG overnight. Bacterial cultures were collected and lysed at 10,000 psi using a French-press in cold-PBS buffer containing protease inhibitor cocktail (Sigma-Aldrich). Bacterial lysates were incubated with GSH-Sepharose beads (GE Amersham) and beads were washed 5 times in PBS before use. MDCK cysts $\left(10^{7}\right)$ grown for $72 \mathrm{~h}$ were washed twice in cold PBS and lysed in $1 \mathrm{ml}$ of TNE buffer
(50 mM Tris, $250 \mathrm{mM} \mathrm{NaCl}, 10 \mathrm{mM}$ EDTA, $\mathrm{pH} 7.4$ ) containing $0.1 \% \mathrm{NP}-40$ and protease inhibitor cocktail (Sigma-Aldrich). Each $1 \mathrm{ml}$ of MDCK cyst lysate was incubated with beads containing $100 \mu \mathrm{g}$ of GST-EpsR (N or C-terminal domains) or GST alone (control) for $2 \mathrm{~h}$. Beads were washed in TNE buffer 5 times, dried by aspiration and eluted in $100 \mu \mathrm{l}$ of Laemmli buffer (LB) and analysed by western blotting. We performed experiments at least three independent times to be confident in the experimental reproducibility.

Co-immunoprecipitation. MDCK cells $\left(10^{7}\right)$ stably expressing PLLP-GFP or EpsR-GFP were grown for $72 \mathrm{~h}$, washed once in cold PBS and lysed in $1 \mathrm{ml}$ of TNE buffer ( $50 \mathrm{mM}$ Tris, $150 \mathrm{mM} \mathrm{NaCl}, 10 \mathrm{mM}$ EDTA, pH 7.4) containing $0.5 \%$ TritonX100 and protease inhibitor cocktail (Sigma-Aldrich). Each $1 \mathrm{ml}$ of MDCK cyst lysate was incubated with $2 \mu \mathrm{g}$ of purified polyclonal anti-GFP (Life Technologies) or $2 \mu \mathrm{g}$ of rabbit antiserum (control). Beads were washed in TNE buffer 5 times, dried by aspiration and bound proteins were eluted in $100 \mu \mathrm{l}$ of LB and analysed by western blotting. We performed experiments at least three independent times to be confident in the experimental reproducibility.

Notch-ligand retroviral transduction. pLZRS-IRES-eGFP retroviral constructs either empty or encoding human Delta-likel or Jagged1 (kindly provided by S. Parreira, Instituto de Histologia e Embriologia, Lisboa, Portugal) were transfected into the packaging 293T Phoenix-Amphotropic cell line (kindly provided by G. Nolan, Stanford University, Stanford, California, USA). Retroviral supernatants obtained from puromycin-selected transfected cells were used for transduction of MDCK and OP9 (ATCC) cells by centrifugation in the presence of Polybrene. Transduced OP9 and MDCK GFP+ cells were isolated by cell sorting (FACSVAntage) $48 \mathrm{~h}$ post-transduction.

Notch activity assays. MDCK cells $\left(10^{6}\right)$ stably expressing murine Notchla-myc were cultured for $48 \mathrm{~h}$ in cysts and western blotting was performed to quantify the cleaved Notchla band at Val 1744 (relative molecular mass 75,000). For transactivation assays on OP9 layers, first OP9 cells were transduced with IRESEGFP, Jagged1-IRES-EGFP or Delta-like1-IRES-EGFP retroviral supernatants prepared in 293-T Phoenix-Ampho cells and FACS-sorted to select a $\mathrm{GFP}^{+}$ population. OP9 cells were passaged with $20 \%$ FBS-supplemented nucleotide-free $\alpha$ MEM (Gibco, Life Technologies). $\mathrm{GFP}^{+} \mathrm{OP} 9$ cells $\left(10^{5}\right)$ were seeded on P35 plates and allowed to attach for $2 \mathrm{~h}$ at $37^{\circ}$ in a $\mathrm{CO}_{2}$-incubator. Then, Notch1MDCK cells $\left(10^{6}\right)$ were seeded on top of the OP9 layer, and grown for $48 \mathrm{~h}$. For MDCK/MDCK transactivation assays, MDCK-II cells were transduced with IRES-EGFP or Jagged1-IRES-EGFP retroviral supernatants and FACS-sorted to select a $\mathrm{GFP}^{+}$population. Notch1-MDCK cells and Jagged1-MDCK cells were mixed 4:1 in $2 \mathrm{ml}$ 2\%MG in MEM (at 100,000 cells $\mathrm{ml}^{-1}$ ) and seeded in Matrigelcoated P35 plates. In all cases, co-cultures were incubated for $48 \mathrm{~h}$ and analysed by western blotting.

Real-time quantitative PCR. Quantitative analysis of gene expression was carried out by real-time quantitative PCR. RNAs were purified from cells or zebrafish guts using the RNAeasy kit (Qiagen) to eliminate genomic DNA contamination. RNA was converted into cDNA using the RNA-to-cDNA High capacity kit and then qPCR was performed using the ABI-PRISM 7900HT SDS system (Applied Biosystems). PCRs were performed on $10 \mathrm{ng}$ template cDNA per well using intron-spanning primers. Specificity was BLAST-analysed for each primer pair, and we performed a melting curve analysis to ensure that a single PCR product was generated.

Morpholino injections. Morpholinos targeting pllp were designed, prepared and injected according to the manufacturer's instructions (Genetools, LLC). We injected $4 \mathrm{ng}$ per one-cell embryo in a 1:1:1 dilution with water and Phenol red. The morpholino sequences were: pllp AUG-MO (MO1): 5' -ACCTTCCCAGGAAAATC CGCCATTT-3' pllp SPL-MO (MO2): 5'-GAATAGTCAAAGAGTCTCACCA CCA-3'.

Statistical analysis. Effect size of 2.4 or greater was expected on the basis of previous publications using the same experimental approaches (that is, acute gene disruption using mutants or RNAi). We chose an appropriate sample size in each experiment to ensure 0.80 statistical power and a significance level of 0.05 . G-Power software was used to predict sample size. Data were then reanalysed post hoc to measure effect size and validate the $t$-tests. In some challenging experiments, certain data sets were not able to reach ideal sample sizes, and we do not provide a $P$ value. We used Student's $t$-test for measuring $P$ values. Significance level was set at 0.05 . For small data sets, normal distribution was predicted from pooled data derived from previous similar experimental approaches (RNAi experiments, cyst formation quantification). For large data sets, we verified normal distribution using $\chi^{2}$. In cases where variance was conspicuously different between two treatments Levene's test was used to verify 
homoscedasticity. Investigators were not blinded to allocation during experiments and outcome assessment.

38. Bosse, F., Hasse, B., Pippirs, U., Greiner-Petter, R. \& Muller, H. W. Proteolipid plasmolipin: localization in polarized cells, regulated expression and lipid raft association in CNS and PNS myelin. J. Neurochem. 86, 508-518 (2003).

39. Schier, A. F. et al. Mutations affecting the development of the embryonic zebrafish brain. Development 123, 165-178 (1996).
40. Doyle, E. L. et al. TAL Effector-Nucleotide Targeter (TALE-NT) 2.0: tools for TAL effector design and target prediction. Nucleic Acids Res. 40, W117-W122 (2012).

41. Rodriguez-Fraticelli, A. E. et al. The Cdc 42 GEF Intersectin 2 controls mitotic spindle orientation to form the lumen during epithelial morphogenesis. J. Cell Biol. 189, 725-738 (2010).

42. Roux, K. J., Kim, D. I., Raida, M. \& Burke, B. A promiscuous biotin ligase fusion protein identifies proximal and interacting proteins in mammalian cells. J. Cell Biol. $196,801-810$ (2012). 


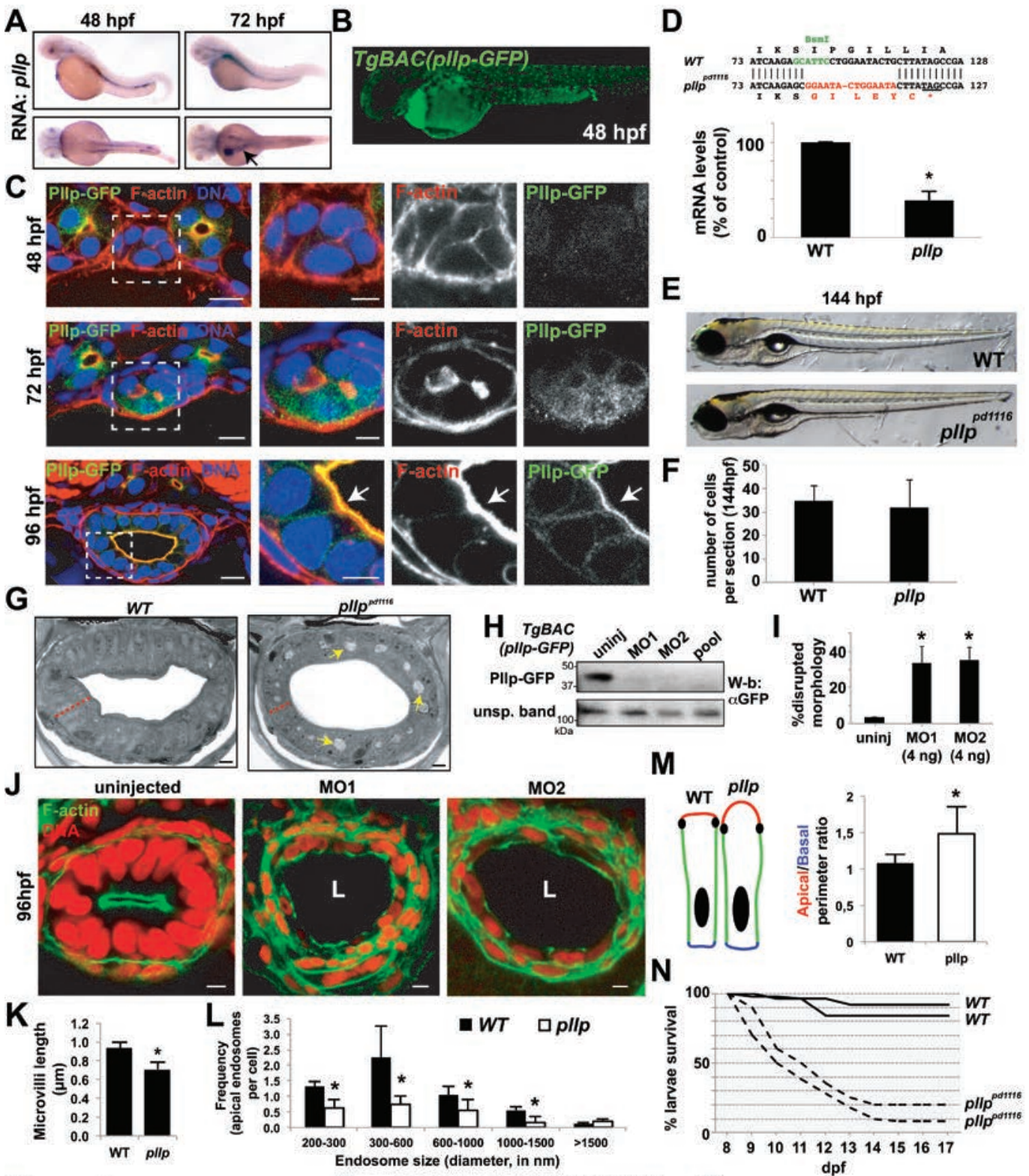

O Phenotype rescue
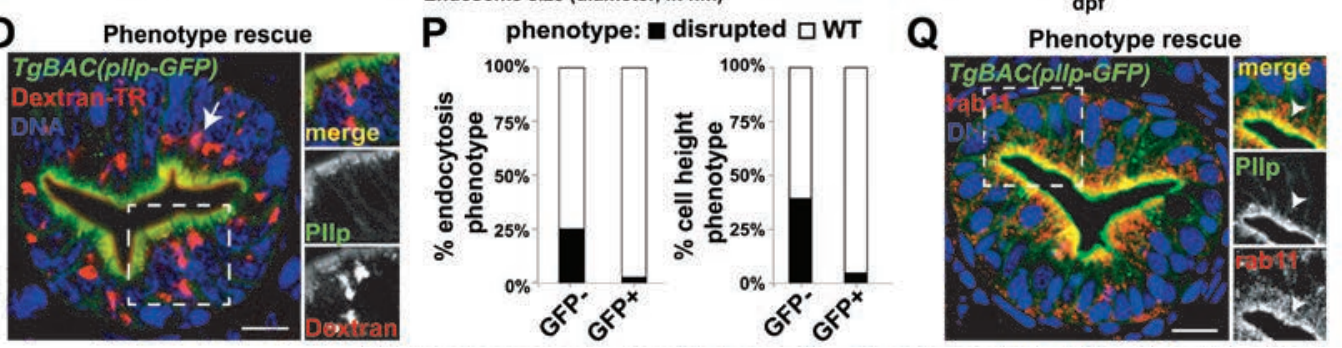
Supplementary Figure 1 Additional characterization of $p / l p$ expression and pllp loss-of-function. (A) In situ hybridization of pllp probe at $48-72 \mathrm{hpf}$. Embryos and larvae were collected and fixed at different time points, and incubated with a DIG-labeled PLLP antisense RNA probe and AP-linked antiDIG antibody. ISH from all different time points were performed at the same time and developed for $2 \mathrm{~h}$ before fixation and cleanup. Arrow indicates gut. (B) $\operatorname{TgBAC}(\mathrm{p} / \mathrm{p}$-GFP) transgenic zebrafish at $48 \mathrm{hpf}$. Transgenic fish were bred and embryos and larvae were analyzed by epifluorescence microscopy. (C) Transverse sections of TgBAC(p/lp-GFP) fish at 48-96-hpf. Sections from the posterior midgut (about $1 / 3$ total gut length before the cloaca) were stained to analyze GFP expression using phalloidin (which labels F-actin in apical microvilli) and DAPI (for DNA). Arrows indicate apical localization. Scale bars, $10 \mu \mathrm{m}$ (magnification, $5 \mu \mathrm{m}$ ). (D) TALEN-generated p/lppd1116 mutant null allele. TALENs were generated to target the first exon of zebrafish $p / l p$ and injected into 1-cell embryos. After raising the founders, we cloned an allele, pd1116 that harbors a null mutation. We confirmed RNA nonsense mediated decay of the $p / / p$ mRNA in the homozygous mutant embryos by measuring $p / l p$ mRNA levels in $p / / p^{p d 1116}$ mutant larvae at 120 hp by RT-qPCR. Results are mean \pm SD \% expression relative to control and normalized with rRNA 18S expression ( $n=3$ RNA extracts; * $p<0.05$ (Student's t test); Statistic source data can be found in Supplementary table 3). (E) Live whole-mount images of WT and p/lppd1116 larvae at $144 \mathrm{hpf}$ (F) Quantification of total number of intestinal cells per section of $6 \mathrm{dpf} p / / p$ pd1116 larvae. $144 \mathrm{hpf}$ larvae were fixed, sectioned and stained with DAPI and $\mathrm{F}$-actin to quantify the total number of intestinal cells per section. Results are represented as mean \pm SD total number of nuclei in a $2 \mu \mathrm{m}$-thick cross-sections ( $\mathrm{n}=10$ sections from 5 WT and 5 p/lp pd1116 fish selected from 3 independent experiments). (G) Toluidin-stained EM sections of p/lppd1116 mutant larvae (400x magnification). Yellow arrows indicate enlarged immature endocytic compartments. Red bars are shown to compare the difference in cell height. Scale bars, $5 \mu \mathrm{m}$. (H) Silencing of TgBAC(pllp-GFP) in morpholino-injected $4 \mathrm{dpf}$ larvae. TgBAC(p/lp-GFP) embryos were injected with MO1 and MO2 morpholinos at one-cell stage and allowed to grow until 4dpf. Whole animal lysates from 20 larvae were analyzed by Western blot, using anti-GFP antibodies. (I) Quantification of \% of morpholino-injected larvae presenting disrupted epithelial columnar organization. WT embryos were injected with $\mathrm{MO} 1$ and $\mathrm{MO} 2$ morpholinos at one-cell stage and grown until 4dpf. Posterior gut sections were stained with Phalloidin (green) and DAPI (red) and analyzed by confocal microscopy. Data are presented as \% of total larvae with disrupted columnar organization ( $n=58$ control, $25 \mathrm{MO} 1$ and $20 \mathrm{MO} 2$-injected larvae pooled from three independent morpholino injection experiments; * $\mathrm{p}<0.05$ (Student's t test)). (J) Confocal images showing the phenotype of morpholino-injected larvae quantified in (I). L, lumen. Scale bars, $5 \mu \mathrm{m}$. (K) Quantification of microvilli length measured in TEM at 6000x magnification. Results are represented as mean \pm SD length (in $\mu \mathrm{m})$ ( $n=20$ microvilli length averages per cell from 5 wt and 5 mutant cells, randomly selected from 2 independent experiments; ${ }^{*} p<0.05$ (Student's t test)). (L) Quantification of apical endosomes in WT and pllppd1116 larvae. Diameters of apical endosomes (within 0-3 $\mu \mathrm{m}$ from the apical surface) were measured from TEM images. Results are represented as mean \pm SD number of endosomes per cell ( $n=10$ cells from 5 wt and 5 mutant fish, randomly selected from 2 independent experiments; * $p<0.05$ (Student's t test)). (M) Quantification of apical membrane expansion in pllppd1116 larvae. Perimeter of the whole luminal apical membrane (red) was divided by the perimeter of the basal membrane (blue). Results are represented as mean $\pm S D$ apical/basal perimeter ratios per cell $(n=20$ cells per condition, from 4 control and 4 mutant fish, randomly selected from 4 independent experiments; * $p<0.05$ (Student's t test)). (N) Quantification of larvae survival in the first $18 \mathrm{dpf}$. WT and p/lppd1116 5 dpf larvae $(\mathrm{n}=25)$ were raised in $1 \mathrm{~L}$ tanks with limited food supply and were assessed by observing heartbeat every day until $18 \mathrm{dpf}$. Solid lines indicate WT larvae. Dotted lines indicate $p / l p^{p d 1116}$ larvae. (0) Rescue of dextran endocytosis in pllppd1116 larvae carrying the TgBAC(p/lp-GFP) pd1115 transgene. $144 \mathrm{hpf}$ larvae were gavaged with Dextran-TR, incubated for $2 \mathrm{~h}$, sectioned and stained with DAPI (blue, DNA). Scale bars, $10 \mu \mathrm{m}$. (P) Quantification of phenotype rescue. $144 \mathrm{hpf}$ larvae from a cross of $\operatorname{TgBAC}\left(\mathrm{p} / \mathrm{l}\right.$-GFP) $\mathrm{p} / \mathrm{l} \mathrm{p}^{+/}$ pd1116 with $p / / p^{p d 1116 / p d 1116}$ fish were gavaged with Dextran-TR, incubated for $2 \mathrm{~h}$, sorted by transgenic GFP expression, and then the percentage of larvae with disrupted endocytosis $(<50 \%)$ and disrupted cell height $(<15 \mu \mathrm{m})$ phenotypes were quantified in each case (GFP+, N=39; GFP-, $\mathrm{N}=85$ ). (Q) Rescue of Rab11 subapical localization in p/lppd116 larvae carrying the TgBAC(p/lp-GFP) transgene. 144hpf WT larvae were fixed, sectioned and stained with the anti-Rab11 antibody (red) and DAPI (blue, DNA). Scale bars, $10 \mu \mathrm{m}$. 
A

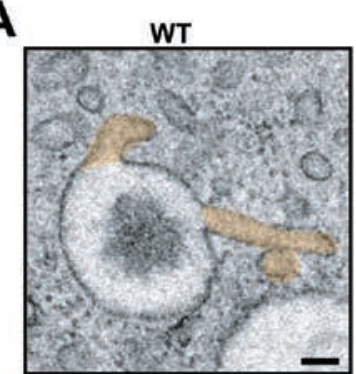

D
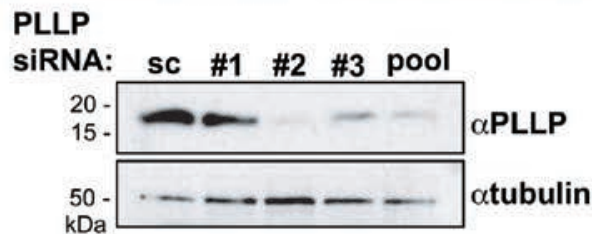

E
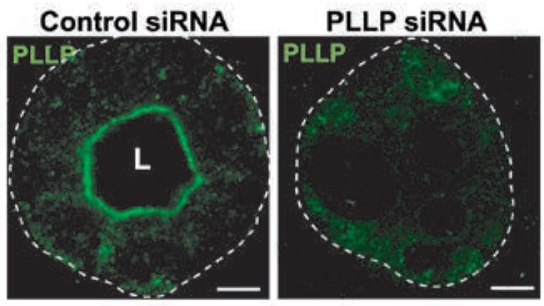

$\mathbf{F}$

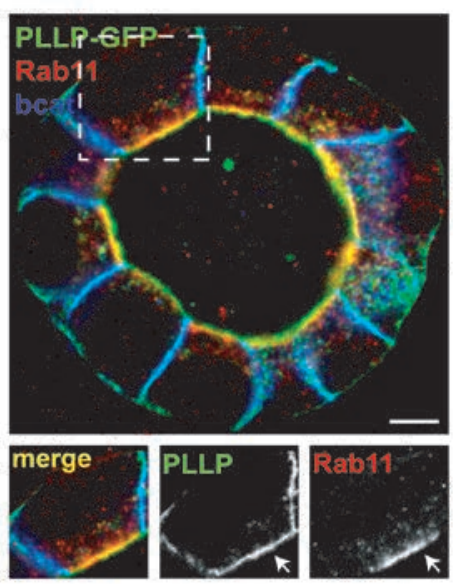

G

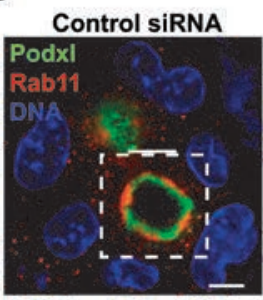

PLLP siRNA

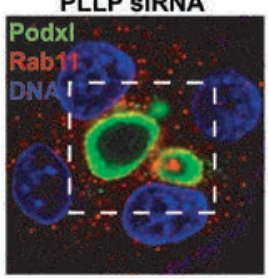

B

Mouse small intestine villus

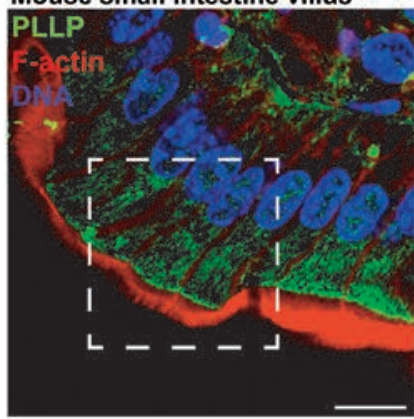

C Mouse Medular kidney tubule

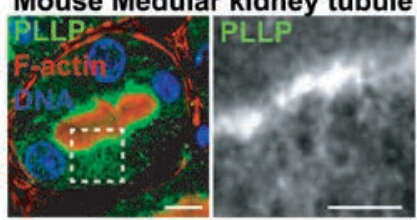

Mouse Cortical kidney tubule
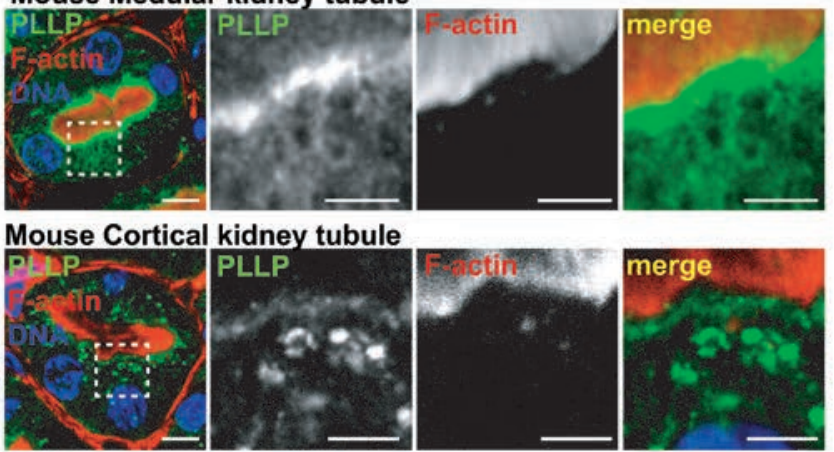

H Pllp-GFp overexp I $15 \mathrm{~nm}$ gold

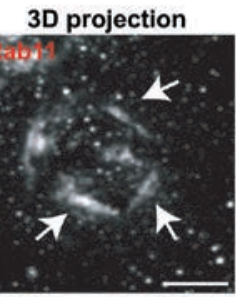

3D projection
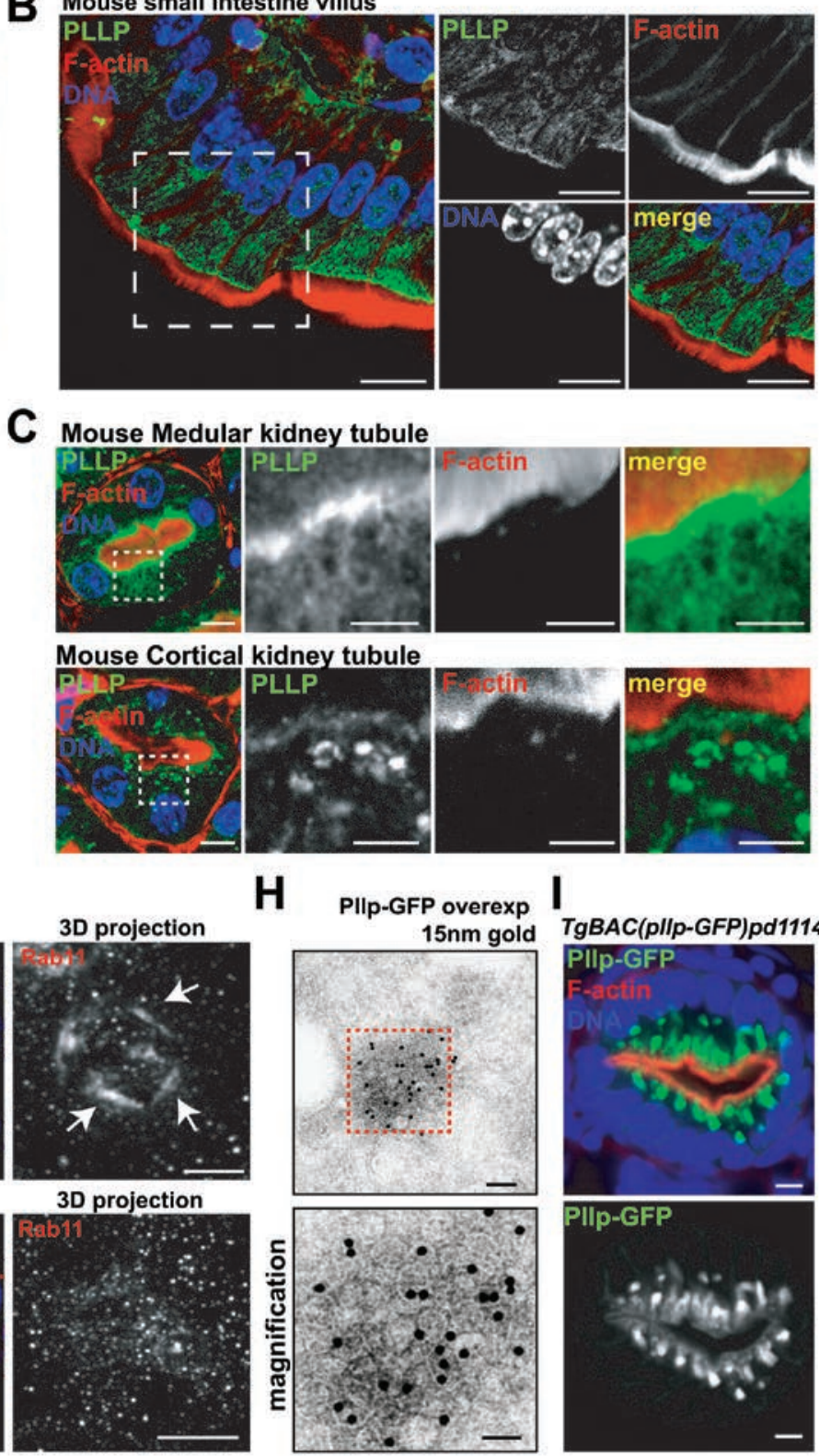

$\operatorname{Tg}$
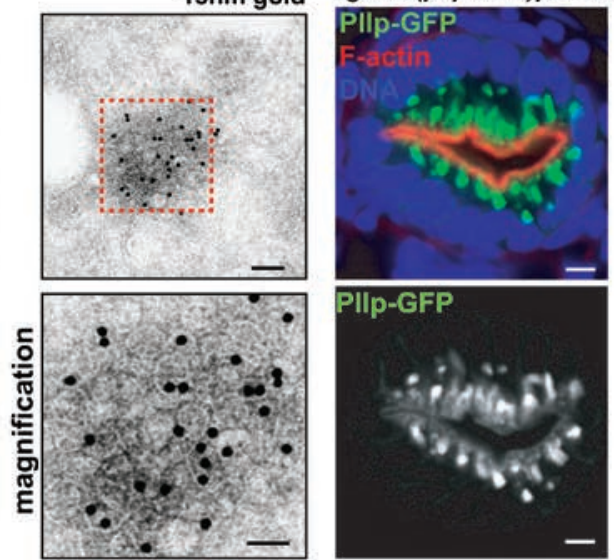

Supplementary Figure 2 PLLP is required for epithelial morphogenesis in 3D-MDCK. (A) Tubular sorting endosomes in apical endosomes in WT and p/lppd1116 larvae. $144 \mathrm{hpf}$ larval gut sections were analyzed by TEM and the number of connected tubular structures as depicted in the micrograph (orange) was quantified. Scale, $100 \mathrm{~nm}$. (B) Localization of endogenous PLLP in mouse intestinal cells. Agarose-embedded sections of 1 month-old BL6 mouse small intestines were stained with the PLLP antibody (green), Phalloidin (red) and DAPI (blue, DNA). A magnification of cells from a villar tip is shown. Scale bars, $10 \mu \mathrm{m}$. (C) Localization of endogenous PLLP in mouse kidney cells. Agarose-embedded sections of 1 month-old BL6 mouse kidneys were stained with the PLLP antibody (green), Phalloidin (red) and DAPI (blue, DNA). Magnifications of a medullar and a cortical kidney tubule are shown. Scale bars, $5 \mu \mathrm{m}$ (magnification, $2 \mu \mathrm{m}$ ). (D) Silencing of PLLP expression in MDCK cysts. MDCK cells transfected with control or PLLPspecific siRNAs were grown to form cysts and lysed after $72 \mathrm{~h}$. Westernblot analysis was performed to quantify PLLP protein levels using control or different PLLP-specific siRNAs oligos. (E) Phenotype of PLLP-KD in MDCK cysts. MDCK cells transfected with control or PLLP-specific siRNAs were grown to form cysts and fixed after $72 \mathrm{~h}$. MDCK cysts were labeled with antiPLLP antibody (green) and analyzed by confocal microscopy with DIC. Notice the disruption of PLLP antibody signal in PLLP-KD cysts. Scale bars, $5 \mu \mathrm{m}$. (F) PLLP and Rab11a colocalization in MDCK cysts. MDCK cells stably expressing PLLP-GFP were grown to form cysts and fixed after 72h. MDCK cysts were labeled with anti-Rab11 antibody (red), anti- $\beta$ catenin (blue) and analyzed by confocal microscopy. Arrow indicates apical recycling endosome. Scale bars, $5 \mu \mathrm{m}$. (G) Rab11 localization in PLLP-KD cysts. MDCK cells transfected with control or PLLP-specific siRNAs were grown to form cysts and fixed after $72 \mathrm{~h}$. MDCK cysts were labeled with anti-Rab11 antibody (red), anti-PodxI (green) DAPI (DNA, blue) and analyzed by confocal microscopy with DIC. Right panels show whole z-axis projection of the Rab11 signal surrounding a single lumen. Arrows indicates apical recycling endosome. Scale bars, $5 \mu \mathrm{m}$. (H) Subcellular EM localization of PLLP-GFP aggregates in overexpressing cells. PLLP-GFP overexpressing cells were FACS-sorted at $48 \mathrm{~h}$, pelleted, seeded into monolayers, and fixed after $24 \mathrm{~h}$. Then, cell pellets were processed for immunogold-EM using anti-GFP and $15 \mathrm{~nm}$ protein-A gold beads. Scale bars, $200 \mathrm{~nm}$ (magnification, $100 \mathrm{~nm}$ ). (I) In vivo overexpression of Pllp-GFP in TgBAC(p/lp-GFP)pd1114 at $5 \mathrm{dpf}$. Posterior gut sections of $5 \mathrm{dpf}$ larvae carrying a TgBAC(p/lp-GFP)pd1114 overexpressing allele were fixed and stained with Phalloidin (red) and DAPI (blue, DNA). Scale bars, $5 \mu \mathrm{m}$. 
A

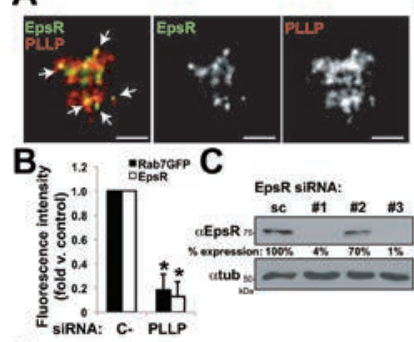

D

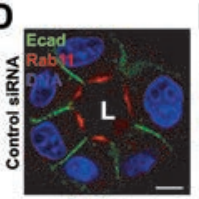

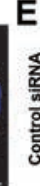

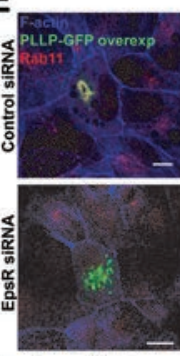

GProximity Ligation Assay (PLA)

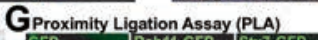

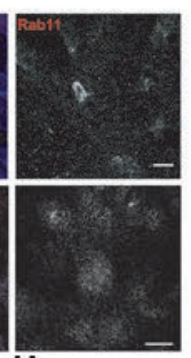

H.
$\mathbf{F}_{\text {Mouse small intestine villus }}$

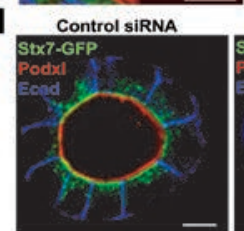

L
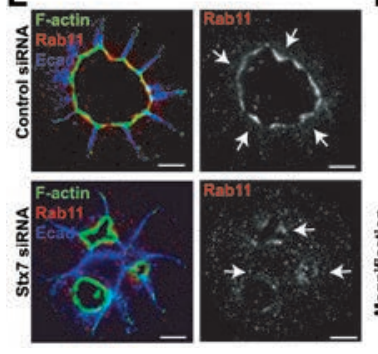

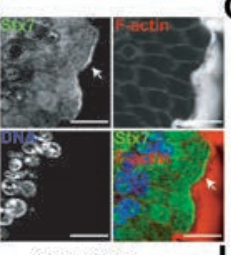

StX7 SIRNA

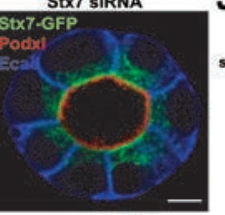

M

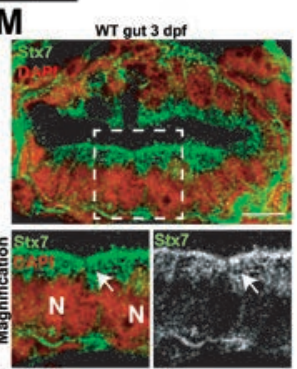

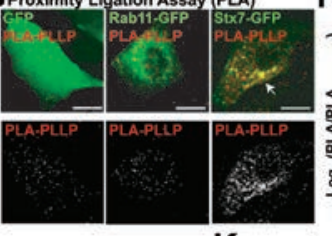

$\mathbf{K}$
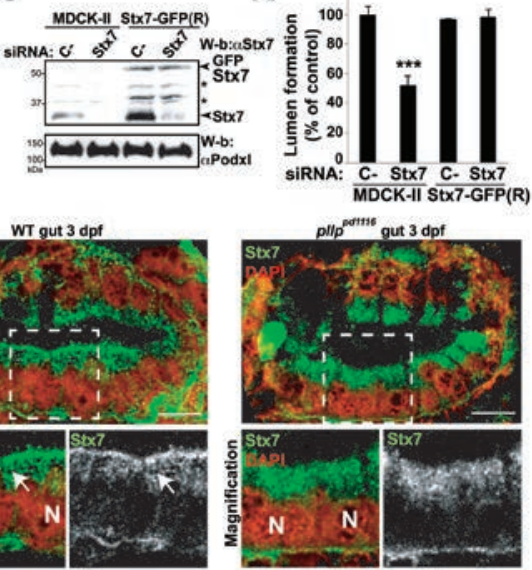

Supplementary Figure 3 EpsR and Stx7 are required for epithelial morphogenesis in 3D-MDCK. (A) EpsR localization in PLLP-Cherry cells. MDCK cells stably expressing PLLP-Cherry were fixed after $72 \mathrm{~h}$, labeled with anti-EpsR antibody (green) and analyzed by confocal microscopy. An $x-y$ section of the apical region of a single cell is shown. Arrows indicate colocalization of PLLP and EpsR. Scale bars, $2 \mu \mathrm{m}$. (B) Quantification of disrupted Rab7-GFP and EpsR fluorescent staining in PLLP-KD cysts. GFPRab7 MDCK cells were transfected with control or PLLP-specific siRNA, grown to form cysts and fixed after $72 \mathrm{~h}$. MDCK cysts were labeled with antiEpsR and PLLP antibodies and analyzed by confocal microscopy to measure fluorescent intensity. Data represented are fold-reduction values of GFP-Rab7 and EpsR staining compared to control ( $n=25$ control and 25 PLLP-KD cells from 3 independent experiments; ${ }^{*} p<0.05$ (Student's $t$ test)). Only cells with less than $10 \%$ of control PLLP expression were counted. (C) Silencing of EpsR expression using siRNA. MDCK cells were transfected with control or EpsR-specific siRNAs, grown to form cysts and lysed after 72h. Westernblot analysis was performed to quantify endogenous EpsR protein levels. (D) Rab11 localization in EpsR-KD cysts. MDCK cells were transfected with control or EpsR-specific siRNAs, grown to form cysts and fixed after 72h. MDCK cysts were labeled with anti-Rab11 antibody (red), E-cadherin (green) and DNA (blue) and analyzed by confocal microscopy. Arrows indicate dispersed Rab11 endosomes in EpsR-KD cysts. Scale bars, $5 \mu \mathrm{m}$. (E) Requirement of EpsR for oversized Rab11 compartment in PLLP-GFP overexpression phenotype. PLLP-GFP overexpressing cells were co-transfected with control or EpsR siRNAs, grown for 48h, fixed and stained to detect Rab11 (red). Scale bars, $5 \mu \mathrm{m}$. (F) Endogenous subapical Stx7 localization in mouse small intestinal villi. Agarose-embedded sections of 1 month-old BL6 mice small intestines were stained with the Stx7 antibody (green), Phalloidin (red) and DAPI (blue, DNA). A magnification of cells from a villar tip is shown. Scale bars, $20 \mu \mathrm{m}$ (magnification, $10 \mu \mathrm{m}$ ). (G) Stx7 and PLLP probe ligation assay (PLA). MDCK cells stably expressing WT untagged PLLP were transiently transfected with GFP mock, Rab11-GFP or Stx7-GFP plasmids. PLA was performed with mouse anti-GFP (green) and rabbit anti-PLLP antibodies and whole-cell maximum z-stack projections of the PLA signal (red) were analyzed by confocal microscopy Arrow indicates colocalization of PLA signal and Stx7-GFP. Scale bars, $10 \mu \mathrm{m}$. (H) Quantification of PLLP PLA. Integrated density of the PLA fluorescent signal was quantified in 16-bit images of maximum z-stack projections and represented as fold-increase over GFP background signal for GFP-Rab11 and GFP-Stx7. Data are average PLA intensity shown as base-2 logarithm of the signal-to-background ratio ( $n=11$ GFP, 15 GFP-Rab11 and 12 GFP-Stx7 cells selected from 2 independent experiments). (I) Phenotype rescue of Stx7-KD MDCK cysts. MDCK cells stably expressing siRNA resistant Stx7-GFP were transfected with control or Stx7-specific siRNAs, grown to form cysts and fixed after $72 \mathrm{~h}$. MDCK cysts were labeled with anti-Podxl antibody (red) and $\beta$ catenin (blue) and analyzed by confocal microscopy. Scale bars, $5 \mu \mathrm{m}$. (J) Silencing of Stx7 expression using siRNA. WT MDCK cells or MDCK cells stably expressing Stx7-GFP were transfected with control or Stx7specific siRNAs, grown to form cysts and lysed after $72 \mathrm{~h}$. Westernblot analysis was performed to quantify Stx7 protein levels. * Unspecific bands from the antibody. (K) Quantification of lumen formation phenotypes in Stx7-KD MDCK cysts and phenotype rescue. Measurements are normalized to WT MDCK cells (control) and expressed as mean $\pm \mathrm{SD}$ percentage relative to control of single lumen-forming cysts (Control, 100 $\pm 5.3 \%$; Stx7-KD, 52.3 $\pm 6.4 \%$; Control GFPStx7, 97.0 $\pm 0.2 \%$; Stx7-KD GFP-Stx7, 98.4 $\pm 5.0 \%$; $n=3$ independent siRNA experiments; ${ }^{* * *} p<0.005$ (Student's $t$ test); Statistic source data can be found in Supplementary table 3). (L) Rab11 localization in Stx7-KD cysts. MDCK cells were transfected with control or Stx7-specific siRNAs, grown to form cysts and fixed after 72h. MDCK cysts were labeled with anti-Rab11 antibody (red), Phalloidin (green) and E-cadherin (blue) and analyzed by confocal microscopy. Arrows indicate apical recycling endosomes. Scale bars, $5 \mu \mathrm{m}$. (M) Stx7 localization in 44hpf pl/ppd1116 mutant larvae. 96hpf WT or p/lppd1116 mutant larvae were fixed with acetone, sectioned and stained with the anti-Stx7 antibody (green), and DAPI (red, DNA). Arrows indicate apical Stx7 localization. Notice the mislocalized dispersed Stx7 in p/lppd1116 larvae. Scale bars, $10 \mu \mathrm{m}$. 


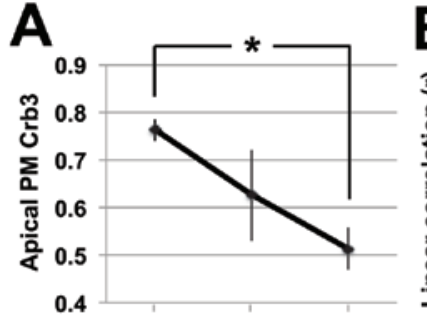

time (h): $\quad 24 \quad 48 \quad 72$

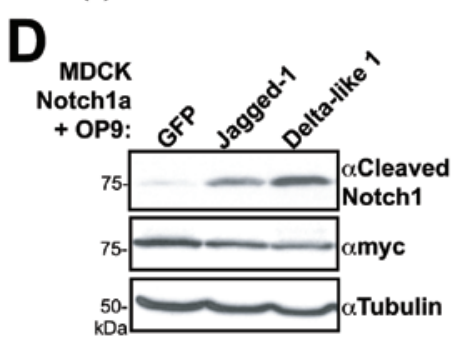

G

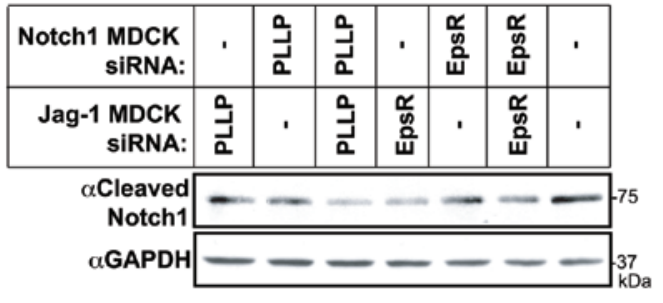

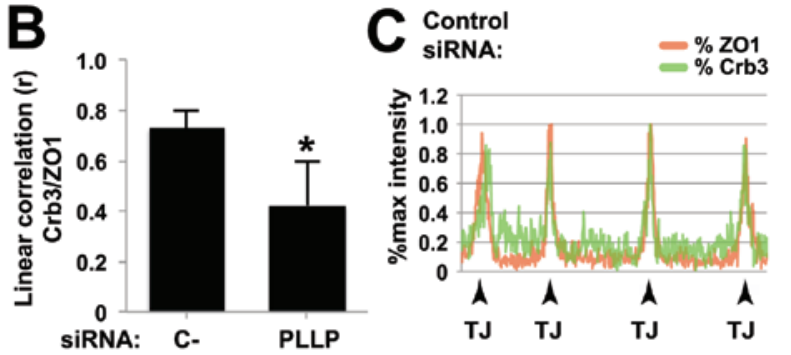

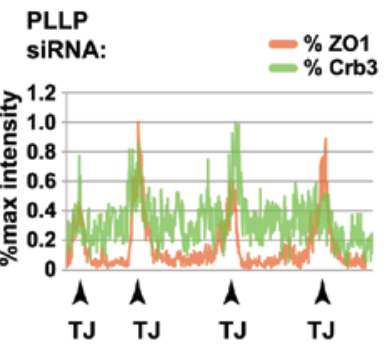

E

\section{Notch/Ligand MDCK coculture model:}

Notch1-expressing MDCK cells

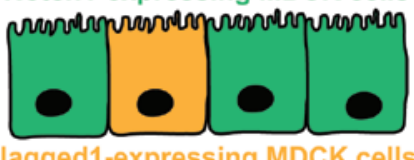

Jagged1-expressing MDCK cells
E

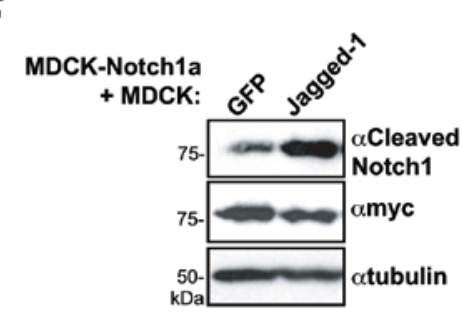

H SiRNA: IC- IPLLP IEPSR

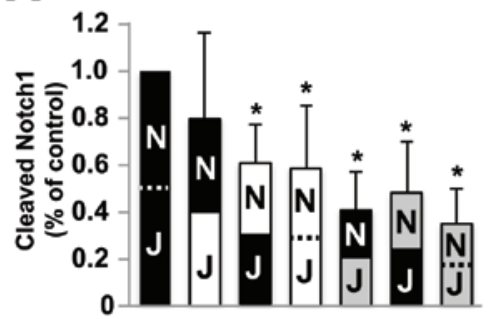

Supplementary Figure 4 Additional analysis on Crb and Notch signaling. (A) Quantification of apical membrane localization of Crb in cysts at different time points. Pearson colocalization coefficients were calculated by staining PodxI and $\mathrm{Crb3}$ at different time points. Data represented are averaged Pearson coefficients $\pm S D$ ( $n=5$ averaged colocalization coefficients from 10 cells per condition, selected from 2 independent experiments; ${ }^{*} p<0.05$ (Student's $t$ test)). (B) Quantification of linear correlation of Crb3 and ZO1 linear profiles. Linear intensity profiles were drawn at the apical plasma membrane and across 4 tight junctions. Data represented are averaged linear (monodimensional) Pearson correlation coefficients $\pm S D$ ( $n=6$ linear profiles taken from 3 cysts per condition, randomly selected from 3 independent siRNA experiments; ${ }^{*} p<0.05$ (Student's $t$ test)). (C) Example ZO1 and Crb3 linear profiles of control-transfected and PLLP siRNA-transfected cysts. Red line, ZO1; green line, Crb3. Data are represented as \% of maximum signal intensity (for each channel). TJ, position of tight junction (determined by peak in ZO1 staining). (D) Western blot of ligand-mediated Notch-1a transactivation assays in MDCK-II/OP9 cocultures. MDCK cells stably expressing Notchla-myc are polarized over a monolayer of mesenchymal OP9 cells stably expressing GFP or Jagged-1 or Delta-like 1. Activated Notch1a is quantified using anti-Cleaved Notchla (Val1744). Tubulin is used as loading control. (E) MDCK coculture system to analyze Notch 1 transactivation by Jagged-1. Stable MDCK cell lines expressing Notch 1 and Jagged-1 are mixed (4:1) and seeded to grow polarized cysts for 48h. (F) Western blot of ligand-mediated Notch-1a transactivation assays in MDCK cocultures. MDCK cells stably expressing Notchla-myc are mixed (4:1) with GFP or Jagged-1 expressing MDCK cells and grown in cysts $(100,000$ cells $/ \mathrm{ml})$ for $48 \mathrm{~h}$. Activated Notchla is quantified using anti-Cleaved Notch1a (Val1744). Tubulin is a loading control. (G) Effect of PLLP-KD or EpsR-KD in Notch1 transactivation by Jagged-1. MDCK cells stably expressing Notch 1 or Jagged-1 were transfected with control, PLLP or EpsR siRNA, mixed as indicated and seeded to grow polarized cysts for $48 \mathrm{~h}$. Cleaved Notch 1 protein levels were analyzed by Western blot. GAPDH is a loading control. (H) Quantification of Notch1 transactivation by Jagged-1 upon PLLP-KD or EpsR-KD. Bars are color coded to indicate control (black), PLLP (white) or EpsR (grey) silencing in either Notch (N) or Jagged-1 (J) MDCK cells. Data are expressed as mean \pm SD as $\%$ of control ( $n=5$ extracts from 5 independent experiments; ${ }^{*} p<0.05$ (Student's $t$ test)). 


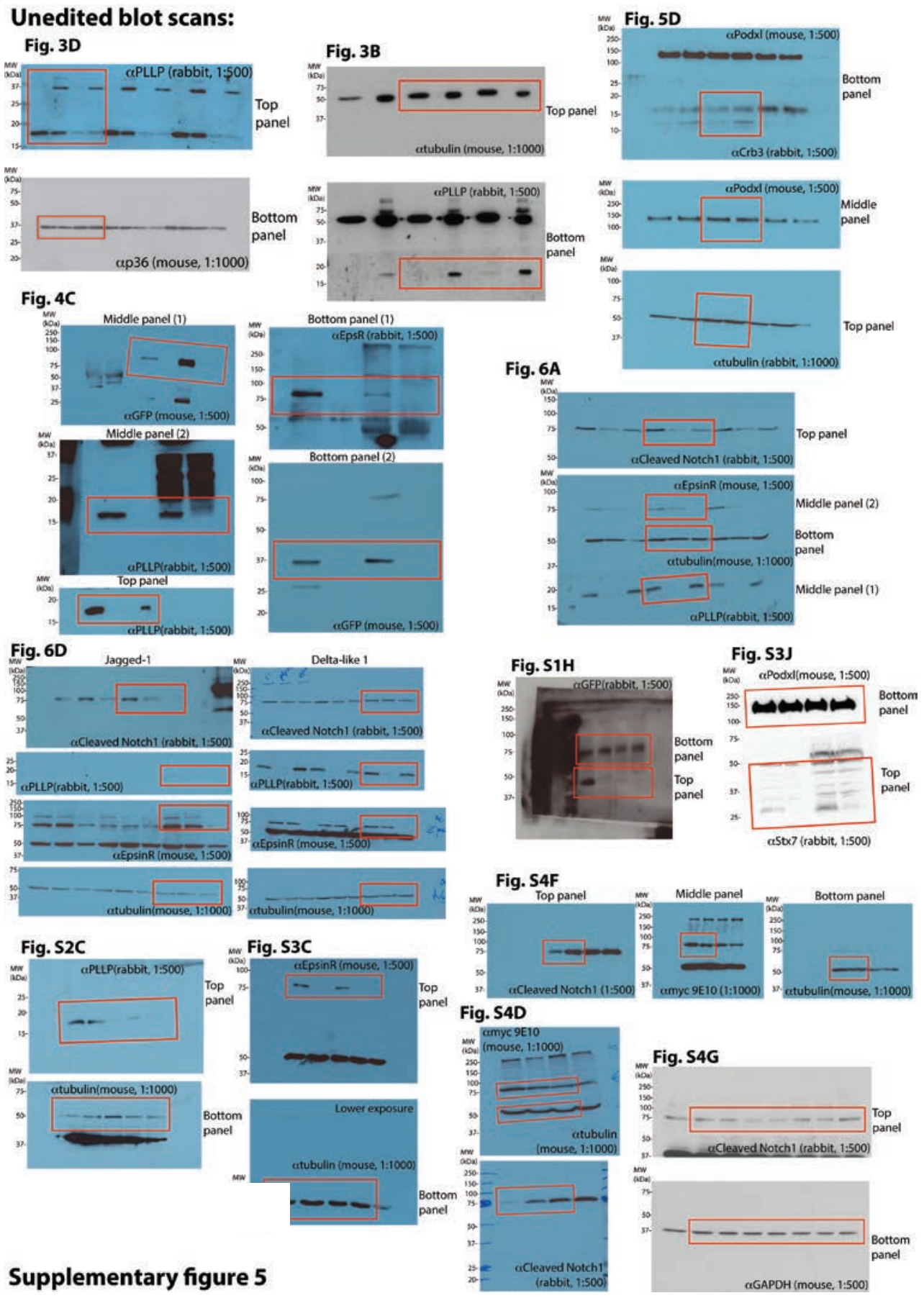

Supplementary Figure 5 Unedited Western-blot scans. Cropped regions are indicated with a red rectangule as appropriate. Antibodies and concentrations are provided for each Western-blot. 


\section{Supplementary Video Legends}

Supplementary Video 1 Stx7/PLLP FRAP experiment. MDCK cells stably expressing PLLP-Cherry were transfected with Stx7-GFP and imaged using confocal microscopy every $4 \mathrm{~s}$. The outlined region of interest was photobleached using the $488 \mathrm{~nm}$ and $564 \mathrm{~nm}$ lasers for 12 rounds after the $4^{\text {th }}$ frame. Frame rate, $10 \mathrm{~s}^{-1}$.

Supplementary Video 2 Crb3 RUSH in MDCK cysts. MDCK cells stably expressing Streptavidin-KDEL and EGFP-SBP-Crb3a were cultured to grow cysts for $72 \mathrm{~h}$. Biotin was added at $\mathrm{t}=0$ and cysts were imaged using confocal microscopy (pinhole $=1 \mu \mathrm{m}$ ) every 1 minute. Frame rate, $3 \mathrm{~s}^{-1}$.

Supplementary Video 3 Crb3 RUSH in control cells. MDCK cells stably expressing Streptavidin-KDEL and EGFP-SBP-Crb3a were transfected with control siRNA and cultured to grow cysts for $72 \mathrm{~h}$. Biotin was added at $\mathrm{t}=0$ and cysts were imaged using confocal microscopy (pinhole $=1 \mu \mathrm{m}$ ) every 1 minute. Frame rate, $3 \mathrm{~s}^{-1}$.

Supplementary Video 4 Crb3 RUSH in PLLP-KD cells. MDCK cells stably expressing Streptavidin-KDEL and EGFP-SBP-Crb3a were transfected with PLLPspecific siRNA and cultured to grow cysts for $72 \mathrm{~h}$. Biotin was added at $\mathrm{t}=0$ and cysts were imaged using confocal microscopy (pinhole $=1 \mu \mathrm{m}$ ) every 1 minute. Frame rate, $3 \mathrm{~s}^{-1}$.

Supplementary Video 5 Crb3 RUSH in Dynasore-treated cells. MDCK cells stably expressing Streptavidin-KDEL and EGFP-SBP-Crb3a were cultured to grow cysts for $72 \mathrm{~h}$. Biotin was added at $\mathrm{t}=0$ and cysts were imaged using confocal microscopy (pinhole $=1 \mu \mathrm{m}$ ) every 1 minute. Dynasore was added at $50 \mu \mathrm{M}$ after $40 \mathrm{~min}$. Frame rate, $3 \mathrm{~s}^{-1}$.

\section{Supplementary Table Legends}

Supplementary Table 1 In vivo biotinylation identification of EpsR as a PLLP-proximal protein (biolD). List of purified peptides from EpsR protein isolated during PLLP-biolD. PLLP-biolD was performed in 3D-MDCK cultures and analyzed by LC-MS/MS peptide mass fingerprinting considering lysine biotinylation as a possible modification. EpsR (Uniprot: Q14677) displayed 4 biotinylated peptides.

Supplementary Table 2 Full list of PLLP-proximal proteins (biolD). Full list of proteins purified by PLLP-biolD. The table contains a combined protein list, as well as individual lists containing all the peptides identified in the control and the PLLP-biolD experimental conditions. Protein molecular weights (MW) and isoelectric point $(\mathrm{Pi})$ are indicated, as well as the number of peptides and peptide protein coverage.

Supplementary Table 3 Statistic source data. This file contains the source data used to derive statistics for the quantifications provided in figures $3 \mathrm{~B}$, 3E, $4 \mathrm{~L}$, $5 \mathrm{D}, 6 \mathrm{~A}, 6 \mathrm{E}, 6 \mathrm{H}, \mathrm{S} 1 \mathrm{D}$ and S3K. 